\title{
Minimization variational principles for acoustics, elastodynamics, and electromagnetism in lossy inhomogeneous bodies at fixed frequency
}

\author{
Graeme W. Milton \\ Department of Mathematics, University of Utah, Salt Lake City UT 84112, USA \\ Pierre Seppecher and Guy Bouchitté \\ Institut de Mathématiques de Toulon, \\ Université de Toulon et du Var, BP 132-83957 La Garde Cedex, France
}

\begin{abstract}
The classical energy minimization principles of Dirichlet and Thompson are extended as minimization principles to acoustics, elastodynamics and electromagnetism in lossy inhomogeneous bodies at fixed frequency. This is done by building upon ideas of Cherkaev and Gibiansky, who derived minimization variational principles for quasistatics. In the absence of free current the primary electromagnetic minimization variational principles have a minimum which is the time-averaged electrical power dissipated in the body. The variational principles provide constraints on the boundary values of the fields when the moduli are known. Conversely, when the boundary values of the fields have been measured, then they provide information about the values of the moduli within the body. This should have application to electromagnetic tomography. We also derive saddle point variational principles which correspond to variational principles of Gurtin, Willis, and Borcea.
\end{abstract}

Keywords: variational principles, acoustics, elastodynamics, electromagnetism

\section{Introduction}

As our goal is to extend the classical minimization principles to acoustics, elastodynamics, and electromagnetism, let us begin by recalling the well-known classical results of electrostatics. Consider the equation of electrostatics

$$
\nabla \cdot \varepsilon \nabla V=0
$$


for the real valued potential $V(\mathbf{x})$ in a body $\Gamma$ containing a locally isotropic material with scalar real positive dielectric constant $\varepsilon(\mathbf{x})$. This equation can be rewritten in the equivalent form

$$
\mathbf{D}=\varepsilon \mathbf{E}, \quad \mathbf{E}=-\nabla V, \quad \nabla \cdot \mathbf{D}=0,
$$

where the first relation is known as the constitutive relation linking the electric displacement field $\mathbf{D}(\mathbf{x})$ and the electrical field $\mathbf{E}(\mathbf{x})$ where these two fields satisfy the differential constraints embodied in the second two relations. Two variational principles are well known. We have the Dirichlet variational principle that

$$
W(V)=\inf _{\underline{V}} W(\underline{V}) \quad \text { where } W(\underline{V})=\int_{\Gamma} \varepsilon|\nabla \underline{V}|^{2} / 2,
$$

in which the infimum is taken over all trial potentials $\underline{V}(\mathbf{x})$ satisfying the boundary condition that $\underline{V}(\mathbf{x})=V(\mathbf{x})$ on the boundary $\partial \Gamma$. We also have dual Thompson variational principle that

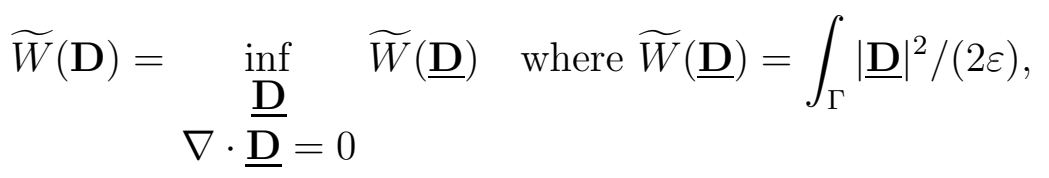

where the infimum is taken over all divergence free trial displacement fields $\underline{\mathbf{D}}$ satisfying the boundary condition that $\underline{\mathbf{D}} \cdot \mathbf{n}=\mathbf{D} \cdot \mathbf{n}$ on $\partial \Gamma$. The quantity $\varepsilon|\nabla V|^{2} / 2=|\mathbf{D}|^{2} /(2 \varepsilon)$ is the electrostatic energy density inside the body, and so these variational principles are also known as energy minimization principles. In the mathematically analogous DC electrical conductivity problem, where $\varepsilon(\mathbf{x})$ and $\mathbf{D}(\mathbf{x})$ are replaced by the conductivity $\sigma(\mathbf{x})$ and the current $\mathbf{J}(\mathbf{x})$, this quantity is half the electrical power dissipation and in that context the variational principles could more accurately be called power dissipation minimization principles.

An appealing feature of these variational principles is that the minimum values $W(V)$ and $\widetilde{W}(\mathbf{D})$ can be expressed in terms of the values of $V(\mathbf{x})$ and $\mathbf{D} \cdot \mathbf{n}$ at the boundary $\partial \Gamma$ :

$$
W(V)=\widetilde{W}(\mathbf{D})=-\frac{1}{2} \int_{\partial \Gamma}(\mathbf{D} \cdot \mathbf{n}) V(\mathbf{x}) .
$$

So these variational principles provide a constraint on the values of $V_{0}(\mathbf{x})=V(\mathbf{x})$ and $q_{0}(\mathbf{x}) \equiv-\mathbf{D}(\mathbf{x}) \cdot \mathbf{n}$ at the boundary $\partial \Gamma$. The relationship between these boundary functions $q_{0}$ and $V_{0}$ is linear and the relationship $q_{0}=\mathbf{N} V_{0}$ defines the Dirichlet to Neumann map $\mathbf{N}$, which is a real self-adjoint positive semi-definite operator. Therefore $\mathbf{N}$ is determined by knowledge of the quadratic form

$$
w\left(V_{0}\right)=\left\langle V_{0}, \mathbf{N} V_{0}\right\rangle / 2,
$$

where for any two scalar or vector, real or complex, valued functions $\mathbf{p}(\mathbf{x})$ and $\mathbf{q}(\mathbf{x})$ defined on the boundary $\partial \Gamma$, we denote

$$
\langle\mathbf{p}, \mathbf{q}\rangle=\int_{\partial \Gamma} \mathbf{p}(\mathbf{x}) \cdot \mathbf{q}(\mathbf{x}) .
$$


Given trial potentials $\underline{V}\left(\mathbf{x}, V_{0}\right)$, parameterized by the function $V_{0}$, with $\underline{V}\left(\mathbf{x}, V_{0}\right)=V_{0}(\mathbf{x})$ on $\partial \Gamma$, the Dirichlet variational principle implies the inequality

$$
w\left(V_{0}\right) \leq W(\underline{V}),
$$

and provides an "upper bound" on the map $\mathbf{N}$. By "upper bound" we mean an upper bound on the associated non-negative quadratic form $w\left(V_{0}\right)$.

To obtain a "lower bound" one uses the Thompson variational principle. Since $w\left(V_{0}\right)=0$ when $V_{0}$ is constant the null-space of $\mathbf{N}$ consists of all constant fields and so $\mathbf{N}$ is determined by knowledge of the quadratic form

$$
\widetilde{w}\left(q_{0}\right)=\left\langle q_{0}, \mathbf{N}^{-1} q_{0}\right\rangle / 2,
$$

for all fields $q_{0}(\mathbf{x})$ with zero average value on $\partial \Gamma$. The functionals $w\left(V_{0}\right)$ and $\widetilde{w}\left(q_{0}\right)$ are Legendre transforms of each other,

$$
w\left(V_{0}\right)=\inf _{q}\left[\left\langle V_{0}, q\right\rangle-\widetilde{w}(q)\right],
$$

where the infimum is over all fields $q(\mathbf{x})$ with zero average value on $\partial \Gamma$, and so an upper bound on the functional $\widetilde{w}(q)$ provides a lower bound on $w\left(V_{0}\right)$. Given trial displacement fields $\underline{\mathbf{D}}\left(\mathbf{x}, q_{0}\right)$ parameterized by the function $q_{0}$, with $-\underline{\mathbf{D}}(\mathbf{x}) \cdot \mathbf{n}=q_{0}(\mathbf{x})$ on $\partial \Gamma$, the Thompson variational principle implies the inequality

$$
\widetilde{w}\left(q_{0}\right) \leq \widetilde{W}(\underline{\mathbf{D}}),
$$

and provides a "lower bound" on the map $\mathbf{N}$.

Thus the Dirichlet and Thompson variational principles allow one to bound the Dirichlet to Neuman map N. As shown by Berryman and Kohn (1990) they can also be used in an inverse fashion: if $\mathbf{N}$ and hence the functionals $w\left(V_{0}\right)$ and $\widetilde{w}\left(q_{0}\right)$ are known one can obtain information about the permittivity distribution $\varepsilon(\mathbf{x})$ from the inequalities (1.8) and (1.11) by appropriately chosing the trial potentials $\underline{V}\left(\mathbf{x}, V_{0}\right)$ and trial displacement fields $\underline{\mathbf{D}}\left(\mathbf{x}, q_{0}\right)$. It has been proved that knowledge of $\mathbf{N}$ uniquely determines $\varepsilon(\mathbf{x})$ when $\varepsilon(\mathbf{x})$ is piecewise analytic (Kohn and Vogelius 1984), or more generally when $\varepsilon(\mathbf{x})$ is smooth (Sylvester and Uhlmann 1987).

In this paper we extend these variational principles to acoustics, elastodynamics, and electromagnetism, in lossy inhomogeneous bodies at fixed frequency. By lossy we mean that the time-averaged dissipation of mechanical or electrical energy into heat is positive everywhere, which means that the imaginary part of certain tensors, such as the permittivity tensor, are positive definite. Like the Dirichlet and Thompson variational principles, the variational principles we obtain are minimization (not saddle point) variational principles. Other variational principles which extend the Dirichlet and Thompson variational principles to dynamics, not assuming time harmonicity, were derived for elastodynamics and electromagnetism by Gurtin (1964) and Willis (1981, 1984). These are minimizing variational principles in the Laplace domain but not in the frequency domain. 
We will recover the Gurtin-Willis variational principles in the time harmonic setting where they correspond to saddle point variational principles. We remark that Ben-Amoz (1966) and Willis (1981, 1984) derived variational principles which are dynamic analogs of the Hashin-Shtrikman (1962b, 1962a, 1962c, 1963) variational principles, involving trial polarizations fields. We do not consider such variational principles here.

Of course given a (inhomogeneous) linear differential operator $\mathcal{A}$, mapping $m$-component complex vector fields to $m$-component complex vector fields, there is a trivial minimization variational principle associated with the equation $\mathcal{A} \mathbf{u}=\mathbf{h}$ for the $m$-component potential $\mathbf{u}$, in which $\mathbf{h}$ is a $m$-component source term. When the equation $\mathcal{A} \mathbf{u}=\mathbf{h}$ with $\mathbf{u}=\mathbf{u}_{0}$ on $\partial \Gamma$ has a unique solution for $\mathbf{u}$, the infimum in

$$
0=\inf _{\underline{\mathbf{u}}} \int_{\Gamma} \overline{(\mathcal{A} \underline{\mathbf{u}}-\mathbf{h})} \cdot(\mathcal{A} \underline{\mathbf{u}}-\mathbf{h})
$$

where the overline denotes complex conjugation and the infimum is over all fields $\underline{\mathbf{u}}$ with $\underline{\mathbf{u}}=\mathbf{u}_{0}$ on $\partial \Gamma$, is clearly attained when $\underline{\mathbf{u}}=\mathbf{u}$. What separates such variational principles from those of Dirichlet and Thompson is that, as explained above, the latter variational principles give us useful information on the boundary values of the fields. We desire variational principles with this property. Also, from a mathematical viewpoint, the existance of a solution cannot be deduced from (1.12), and in case there is a solution no interesting inequality results from (1.12).

In an important development Cherkaev and Gibiansky (1994) extended the Dirichlet and Thompson variational principles to the quasistatic electromagnetic equations (and also to the quasistatic elastodynamic equations) where the equations (1.1) and (1.2) still hold but $\varepsilon(\mathbf{x}), V(\mathbf{x}), \mathbf{E}(\mathbf{x})$ and $\mathbf{D}(\mathbf{x})$ are complex, and the imaginary part of $\varepsilon(\mathbf{x})$ is positive. Since we build upon their work let us briefly review their ideas in this context.

By rewriting the constitutive law $\mathbf{D}=\varepsilon \mathbf{E}$ in terms of its real and imaginary parts

$$
\left(\begin{array}{c}
\mathbf{D}^{\prime \prime} \\
\mathbf{D}^{\prime}
\end{array}\right)=\left(\begin{array}{cc}
\varepsilon^{\prime \prime} & \varepsilon^{\prime} \\
\varepsilon^{\prime} & -\varepsilon^{\prime \prime}
\end{array}\right)\left(\begin{array}{c}
\mathbf{E}^{\prime} \\
\mathbf{E}^{\prime \prime}
\end{array}\right)
$$

where the prime denotes the real part, while the double prime denotes the imaginary part, it is evident that since $\varepsilon^{\prime \prime}(\mathbf{x})>0$ one has the Cherkaev-Gibiansky saddle point variational principle

$$
Q\left(V^{\prime}, V^{\prime \prime}\right)=\inf _{\underline{V}^{\prime}} \sup _{\underline{V}^{\prime \prime}} Q\left(\underline{V}^{\prime}, \underline{V^{\prime \prime}}\right)=\sup _{\underline{V}^{\prime \prime}} \inf _{\underline{V}^{\prime}} Q\left(\underline{V}^{\prime}, \underline{V^{\prime \prime}}\right)
$$

where

$$
Q\left(\underline{V}^{\prime}, \underline{V}^{\prime \prime}\right)=\int_{G G}\left(\begin{array}{c}
\nabla \underline{V^{\prime}} \\
\nabla \underline{V}^{\prime \prime}
\end{array}\right) \cdot\left(\begin{array}{cc}
\varepsilon^{\prime \prime} & \varepsilon^{\prime} \\
\varepsilon^{\prime} & -\varepsilon^{\prime \prime}
\end{array}\right)\left(\begin{array}{c}
\nabla \underline{V^{\prime}} \\
\nabla \underline{V^{\prime \prime}}
\end{array}\right),
$$

and the infimum and supremum are over trial potentials with $\underline{V^{\prime}}(\mathbf{x})=V^{\prime}(\mathbf{x})$ and $\underline{V^{\prime \prime}}(\mathbf{x})=$ $V^{\prime \prime}(\mathbf{x})$ on the boundary $\partial \Gamma$. To obtain a minimization variational principle from this saddle point variational principle Cherkaev and Gibiansky (1994) make a partial Legendre 
transform of the saddle shaped quadratic form to convert it into a convex quadratic form. This is equivalent to rewriting the constitutive law in the form

$$
\left(\begin{array}{l}
\mathbf{D}^{\prime \prime} \\
\mathbf{E}^{\prime \prime}
\end{array}\right)=\mathcal{E}\left(\begin{array}{c}
\mathbf{E}^{\prime} \\
-\mathbf{D}^{\prime}
\end{array}\right)
$$

where the matrix

$$
\mathcal{E}=\left(\begin{array}{cc}
\varepsilon^{\prime \prime}+\left(\varepsilon^{\prime}\right)^{2} /\left(\varepsilon^{\prime \prime}\right) & \varepsilon^{\prime} / \varepsilon^{\prime \prime} \\
\varepsilon^{\prime} / \varepsilon^{\prime \prime} & 1 / \varepsilon^{\prime \prime}
\end{array}\right)
$$

is positive definite when $\varepsilon^{\prime \prime}(\mathbf{x})$ is positive. From this it is evident that one has the Cherkaev-Gibiansky minimization variational principle:

$$
Y\left(V^{\prime}, \mathbf{D}^{\prime}\right)=\inf _{\underline{V}^{\prime}} \inf _{\nabla} \quad Y\left(\underline{\mathbf{D}}^{\prime}, \underline{\mathbf{D}}^{\prime}\right),
$$

where

$$
Y\left(\underline{V^{\prime}}, \underline{\mathbf{D}}^{\prime}\right)=\int_{\Gamma}\left(\begin{array}{c}
\nabla \\
\underline{\mathrm{D}}^{\prime}
\end{array}\right) \cdot \mathcal{E}\left(\begin{array}{c}
\nabla \\
{\underline{V^{\prime}}}^{\prime}
\end{array}\right),
$$

and the infimums are over all trial real potentials with $\underline{V}^{\prime}(\mathbf{x})=V^{\prime}(\mathbf{x})$ on $\partial \Gamma$, and all trial real divergence free displacement fields with $\underline{\mathbf{D}} \cdot \mathbf{n}=\mathbf{D} \cdot \mathbf{n}$ on $\partial \Gamma$.

As an aside we remark that, as shown in section 18 of Milton (1990), one can extend these ideas of Gibiansky and Cherkaev to obtain variational principles for problems where the constitutive law takes the form $\mathbf{D}=\varepsilon \mathbf{E}$, where the matrix valued field $\varepsilon(\mathbf{x})$ is not symmetric. For example, for conduction in a fixed magnetic field, the conductivity tensor $\boldsymbol{\sigma}(\mathbf{x})$ is real but not symmetric. Expressing $\boldsymbol{\sigma}(\mathbf{x})=\boldsymbol{\sigma}_{s}(\mathbf{x})+\boldsymbol{\sigma}_{a}(\mathbf{x})$, where $\boldsymbol{\sigma}_{s}(\mathbf{x})=\boldsymbol{\sigma}_{s}^{T}(\mathbf{x})$ and $\boldsymbol{\sigma}_{a}(\mathbf{x})=-\boldsymbol{\sigma}_{a}^{T}(\mathbf{x})$ are the symmetric and antisymmetric parts of the conductivity tensor, one considers current fields $\mathbf{J}(\mathbf{x})$ and electric fields $\mathbf{E}(\mathbf{x})$ which solve the conductivity equations

$$
\mathbf{J}=\left(\boldsymbol{\sigma}_{s}+\boldsymbol{\sigma}_{a}\right) \mathbf{E}, \quad \mathbf{E}=-\nabla V, \quad \nabla \cdot \mathbf{J}=0,
$$

in conjunction with current fields $\widehat{\mathbf{J}}(\mathbf{x})$ and electric fields $\widehat{\mathbf{E}}(\mathbf{x})$ which solve the adjoint problem

$$
\widehat{\mathbf{J}}=\left(\boldsymbol{\sigma}_{s}-\boldsymbol{\sigma}_{a}\right) \widehat{\mathbf{E}}, \quad \widehat{\mathbf{E}}=-\nabla \widehat{V}, \quad \nabla \cdot \widehat{\mathbf{J}}=0 .
$$

By adding and subtracting the two constitutive laws one gets a new constitutive law

$$
\left(\begin{array}{c}
\mathbf{J}_{s} \\
-\mathbf{J}_{a}
\end{array}\right)=\left(\begin{array}{cc}
\boldsymbol{\sigma}_{s} & \boldsymbol{\sigma}_{a} \\
-\boldsymbol{\sigma}_{a} & -\boldsymbol{\sigma}_{s}
\end{array}\right)\left(\begin{array}{c}
\mathbf{E}_{s} \\
\mathbf{E}_{a}
\end{array}\right),
$$

involving fields

$$
\mathbf{J}_{s} \equiv \mathbf{J}+\widehat{\mathbf{J}}, \quad \mathbf{J}_{a} \equiv \mathbf{J}-\widehat{\mathbf{J}}, \quad \mathbf{E}_{s} \equiv \mathbf{E}+\widehat{\mathbf{E}}, \quad \mathbf{E}_{a} \equiv \mathbf{E}-\widehat{\mathbf{E}}
$$


that satisfy the differential constraints

$$
\nabla \cdot \mathbf{J}_{s}=\nabla \cdot \mathbf{J}_{a}=0, \quad \mathbf{E}_{s}=-\nabla V_{s}, \quad \mathbf{E}_{a}=-\nabla V_{a},
$$

where $V_{s} \equiv V+\widehat{V}$ and $V_{a} \equiv V-\widehat{V}$. Conversely, given any fields which solve (1.22) and (1.24), one can recover the associated fields which solve (1.20) and (1.21). The two problems are equivalent. Since the matrix entering the constitutive law (1.22) is real and symmetric there is clearly an associated saddle point variational principle when $\boldsymbol{\sigma}_{s}(\mathbf{x})$ is positive definite for all $\mathbf{x}$. Fannjiang and Papanicolaou (1994) show how this variational principle provides useful asymptotic estimates for the mathematically analogous problem

of convection enhanced diffusion. Also by rewriting the constitutive law (1.22) in the equivalent form

$$
\left(\begin{array}{c}
\mathbf{J}_{s} \\
\mathbf{E}_{a}
\end{array}\right)=\left(\begin{array}{cc}
\boldsymbol{\sigma}_{s}-\boldsymbol{\sigma}_{a} \boldsymbol{\sigma}_{s}^{-1} \boldsymbol{\sigma}_{a} & \boldsymbol{\sigma}_{a} \boldsymbol{\sigma}_{s}^{-1} \\
-\boldsymbol{\sigma}_{s}^{-1} \boldsymbol{\sigma}_{a} & \boldsymbol{\sigma}_{s}^{-1}
\end{array}\right)\left(\begin{array}{c}
\mathbf{E}_{s} \\
\mathbf{J}_{a}
\end{array}\right)
$$

which involves a matrix which is symmetric and positive definite when $\boldsymbol{\sigma}_{s}(\mathbf{x})$ is positive definite for all $\mathbf{x}$, one obtains an associated minimization variational principle (Milton 1990). In this paper we do not treat such cases where the constitutive laws involve non-symmetric matrices: this will be covered elsewhere.

\section{General Theory}

Let $d$ be the dimension of the space and let $\mathcal{F}(\mathbf{x})$ and $\mathcal{G}(\mathbf{x})$ be two complex valued fields of the form

$$
\mathcal{F}=\left(\begin{array}{c}
\mathbf{F} \\
\mathbf{f}
\end{array}\right), \quad \mathcal{G}=\left(\begin{array}{c}
\mathbf{G} \\
\mathrm{g}
\end{array}\right)
$$

where $\mathbf{F}(\mathbf{x})$ and $\mathbf{G}(\mathbf{x})$ are $m \times d$ dimensional matrix fields and $\mathbf{f}(\mathbf{x})$ and $\mathbf{g}(\mathbf{x})$ are $m$ component vector fields, and which satisfy the differential constraints

$$
\mathcal{F}=\sqcap \mathbf{u}, \quad \mathbf{h}+\sqcup \mathcal{G}=0,
$$

where $\mathbf{u}(\mathbf{x})$ is an $m$-component potential and $\mathbf{h}(\mathbf{x})$ is some $m$-component source term and

$$
\sqcap u \equiv\left(\begin{array}{c}
\nabla \mathbf{u} \\
\mathbf{u}
\end{array}\right), \quad \sqcup \mathcal{G} \equiv-\nabla \cdot \mathbf{G}+\mathbf{g}
$$

serve to define the operators $\sqcap$ and $\sqcup$. The differential constraints imply the key property that on any domain $\Gamma$

$$
\int_{\Gamma} \mathcal{G} \cdot \mathcal{F}+\mathbf{h} \cdot \mathbf{u}=\int_{\partial \Gamma}(\mathbf{G} \cdot \mathbf{n}) \cdot \mathbf{u}
$$

as follows from integration by parts, where

$$
\mathcal{G} \cdot \mathcal{F} \equiv \mathbf{G} \cdot \mathbf{F}+\mathbf{g} \cdot \mathbf{f}, \quad \mathbf{G} \cdot \mathbf{F} \equiv \operatorname{Tr}\left(\mathbf{G}^{T} \mathbf{F}\right),
$$


and $\mathbf{n}$ is the outward unit normal to the surface $\partial \Gamma$. (Note that (2.5) is an inner product only when $\mathcal{G}$ and $\mathcal{F}$ are real, since it does not involve complex conjugation). Here, following standard notation, we define

$$
\{\nabla \mathbf{u}\}_{i j}=\frac{\partial u_{i}}{\partial x_{j}}, \quad\{\nabla \cdot \mathbf{G}\}_{i}=\sum_{j=1}^{d} \frac{\partial G_{i j}}{\partial x_{j}} .
$$

Now suppose the fields $\mathcal{F}$ and $\mathcal{G}$ are linked by the constitutive relation

$$
\mathcal{G}(\mathbf{x})=\mathbf{Z}(\mathbf{x}) \mathcal{F}(\mathbf{x})
$$

where $\mathbf{Z}(\mathbf{x})=\mathbf{Z}^{\prime}(\mathbf{x})+i \mathbf{Z}^{\prime \prime}(\mathbf{x})$ is a complex symmetric linear map (which is represented by a complex symmetric $m(d+1) \times m(d+1)$ matrix when $\mathcal{G}$ and $\mathcal{F}$ are represented by $m(d+1)$ component vectors $)$. The map $\mathbf{Z}$ can be expressed in block form as

$$
\mathbf{Z}=\left(\begin{array}{cc}
\mathbf{L} & \mathbf{K} \\
\mathbf{K}^{T} & \mathbf{M}
\end{array}\right)
$$

where (for each $\mathbf{x}) \mathbf{L}(\mathbf{x})$ is a symmetric linear map on the space of $m \times d$ dimensional matrices, $\mathbf{K}(\mathbf{x})$ is a linear map from $m$ component vectors to $m \times d$ dimensional matrices, $\mathbf{K}^{T}(\mathbf{x})$ is a linear map from $m \times d$ dimensional matrices to $m$ component vectors, and $\mathbf{M}(\mathbf{x})$ is a symmetric linear map on the space of $m$ component vectors. Thus, written in terms of components, (2.7) implies

$$
\begin{aligned}
G_{i j} & =L_{i j k \ell} F_{k \ell}+K_{i j k} f_{k}, \\
g_{i} & =K_{k \ell i} F_{k \ell}+M_{i k} f_{k},
\end{aligned}
$$

in which sums of repeated indices are assumed. Since $\mathbf{L}$ and $\mathbf{M}$ are symmetric maps, we have

$$
L_{i j k \ell}=L_{k \ell i j}, \quad M_{i k}=M_{k i} .
$$

The constitutive law and differential constraints imply that $\mathbf{u}$ satisfies

$$
\mathbf{h}+\sqcup(\mathbf{Z} \sqcap \mathbf{u})=0,
$$

or equivalently,

$$
\nabla \cdot(\mathbf{L} \nabla \mathbf{u}+\mathbf{K u})=\mathbf{h}+\mathbf{K}^{T} \nabla \mathbf{u}+\mathbf{M u}
$$

Our analysis thus applies to equations of this form, with $\mathbf{L}$ and $\mathbf{M}$ having the symmetries (2.10).

Let $(\mathbf{u}, \mathcal{F}, \mathcal{G})$ be a solution of (2.2) and (2.7). Taking real and imaginary parts, the differential constraints imply

$$
\mathcal{F}^{\prime}=\sqcap \mathbf{u}^{\prime}, \quad \mathbf{h}^{\prime}+\sqcup \mathcal{G}^{\prime}=0, \quad \mathcal{F}^{\prime \prime}=\sqcap \mathbf{u}^{\prime \prime}, \quad \mathbf{h}^{\prime \prime}+\sqcup \mathcal{G}^{\prime \prime}=0,
$$


where the prime denotes the real part and the double prime the imaginary part, and the constitutive law can be written in the form

$$
\left(\begin{array}{l}
\mathcal{G}^{\prime \prime} \\
\mathcal{G}^{\prime}
\end{array}\right)=\left(\begin{array}{cc}
\mathbf{Z}^{\prime \prime} & \mathbf{Z}^{\prime} \\
\mathbf{Z}^{\prime} & -\mathbf{Z}^{\prime \prime}
\end{array}\right)\left(\begin{array}{c}
\mathcal{F}^{\prime} \\
\mathcal{F}^{\prime \prime}
\end{array}\right)
$$

To make use of this representation let us further suppose that the imaginary part of $\mathbf{Z}$ satisfies

$$
\mathbf{Z}^{\prime \prime}(\mathbf{x}) \geq \alpha \mathbf{I}, \quad \forall \mathbf{x} \in \Gamma,
$$

for some $\alpha>0$. Extending the ideas of Cherkaev and Gibiansky (1994) consider the real valued quadratic functional

$$
\begin{aligned}
Q\left(\underline{\mathbf{u}}^{\prime}, \underline{\mathbf{u}}^{\prime \prime}\right) & =\int_{\Gamma}\left(\begin{array}{c}
\sqcap \underline{\mathbf{u}}^{\prime} \\
\sqcap \underline{\mathbf{u}}^{\prime \prime}
\end{array}\right) \cdot\left(\begin{array}{cc}
\mathbf{Z}^{\prime \prime} & \mathbf{Z}^{\prime} \\
\mathbf{Z}^{\prime} & -\mathbf{Z}^{\prime \prime}
\end{array}\right)\left(\begin{array}{l}
\sqcap \underline{\mathbf{u}}^{\prime} \\
\sqcap \underline{\mathbf{u}}^{\prime \prime}
\end{array}\right)+2\left(\mathbf{h}^{\prime \prime} \cdot \underline{\mathbf{u}}^{\prime}+\mathbf{h}^{\prime} \cdot \underline{\mathbf{u}}^{\prime \prime}\right) \\
& =\int_{\Gamma}[(\sqcap \underline{\mathbf{u}}) \cdot \mathbf{Z} \sqcap \underline{\mathbf{u}}+2 \mathbf{h} \cdot \mathbf{u}]^{\prime \prime},
\end{aligned}
$$

subject to the boundary conditions that $\underline{\mathbf{u}}^{\prime}=\mathbf{u}^{\prime}$ and $\underline{\mathbf{u}}^{\prime \prime}=\mathbf{u}^{\prime \prime}$ on $\partial \Gamma$. Let $\mathbf{s}(\mathbf{x})$ be a real valued $m$ component vector field with $\mathbf{s}=0$ on $\partial \Gamma$. Now $Q\left(\underline{\mathbf{u}}^{\prime}+\mathbf{s}, \underline{\mathbf{u}}^{\prime \prime}\right)$ is a convex quadratic function of $\mathbf{s}$ (with a linear component) while $Q\left(\underline{\mathbf{u}}^{\prime}, \underline{\mathbf{u}}^{\prime \prime}+\mathbf{s}\right)$ is a concave quadratic function of $\mathbf{s}$ (with a linear component). Therefore on the space of fields satisfying the boundary conditions the pair $\left(\underline{\mathbf{u}}^{\prime}, \underline{\mathbf{u}}^{\prime \prime}\right)$ which are at the saddle point of the variational problem

$$
\inf _{\underline{\mathbf{u}}^{\prime}} \sup _{\underline{\mathbf{u}}^{\prime \prime}} Q\left(\underline{\mathbf{u}}^{\prime}, \underline{\mathbf{u}}^{\prime \prime}\right)
$$

exists and is unique and corresponds to the stationary point of $Q\left(\underline{\mathbf{u}}^{\prime}, \underline{\mathbf{u}}^{\prime \prime}\right)$, and is also at the saddle point of the variational problem

$$
\sup _{\underline{\mathbf{u}}^{\prime \prime}} \inf _{\underline{\mathbf{u}}^{\prime}} Q\left(\underline{\mathbf{u}}^{\prime}, \underline{\mathbf{u}}^{\prime \prime}\right) \text {. }
$$

Now let us prove we have the variational principle

$$
Q\left(\mathbf{u}^{\prime}, \mathbf{u}^{\prime \prime}\right)=\inf _{\underline{\mathbf{u}}^{\prime}} \sup _{\underline{\mathbf{u}}^{\prime \prime}} Q\left(\underline{\mathbf{u}}^{\prime}, \underline{\mathbf{u}}^{\prime \prime}\right)=\sup _{\underline{\mathbf{u}}^{\prime \prime}} \inf _{\underline{\mathbf{u}}^{\prime}} Q\left(\underline{\mathbf{u}}^{\prime}, \underline{\mathbf{u}}^{\prime \prime}\right)
$$

where the infimum and supremum are over fields with $\underline{\mathbf{u}}^{\prime}$ and $\underline{\mathbf{u}}^{\prime \prime}$ with any fixed values $\mathbf{u}_{0}^{\prime}$ and $\mathbf{u}_{0}^{\prime \prime}$ at the boundary $\partial \Gamma$ and $\mathbf{u}^{\prime}$ and $\mathbf{u}^{\prime \prime}$ are the solutions with these prescribed boundary conditions.

Let $(\mathbf{u}, \mathcal{F}, \mathcal{G})$ be a solution of (2.2) and (2.7). As $\mathbf{s}=0$ on $\partial \Gamma$, in a similar way as (2.4) was derived, we get

$$
\int_{\Gamma} \mathcal{G}^{\prime \prime} \cdot(\sqcap \mathbf{s})+\mathbf{h}^{\prime \prime} \cdot \mathbf{s}=0, \quad \int_{\Gamma} \mathcal{G}^{\prime} \cdot(\sqcap \mathbf{s})+\mathbf{h}^{\prime} \cdot \mathbf{s}=0 .
$$


Thus we see that

$$
\begin{aligned}
Q\left(\mathbf{u}^{\prime}+\mathbf{s}, \mathbf{u}^{\prime \prime}\right) & =Q\left(\mathbf{u}^{\prime}, \mathbf{u}^{\prime \prime}\right)+\int_{\Gamma} 2 \mathcal{G}^{\prime \prime} \cdot(\sqcap \mathbf{s})+2 \mathbf{h}^{\prime \prime} \cdot \mathbf{s}+(\sqcap \mathbf{s}) \cdot \mathbf{Z}^{\prime \prime} \sqcap \mathbf{s} \\
& =Q\left(\mathbf{u}^{\prime}, \mathbf{u}^{\prime \prime}\right)+\int_{\Gamma}(\sqcap \mathbf{s}) \cdot \mathbf{Z}^{\prime \prime} \sqcap \mathbf{s},
\end{aligned}
$$

and in the same way we get

$$
Q\left(\mathbf{u}^{\prime}, \mathbf{u}^{\prime \prime}+\mathbf{s}\right)=Q\left(\mathbf{u}^{\prime}, \mathbf{u}^{\prime \prime}\right)-\int_{\Gamma}(\sqcap \mathbf{s}) \cdot \mathbf{Z}^{\prime \prime} \sqcap \mathbf{s} .
$$

So we see that the saddle point of $(2.17)$ is at $\left(\underline{\mathbf{u}}^{\prime}, \underline{\mathbf{u}}^{\prime \prime}\right)=\left(\mathbf{u}^{\prime}, \mathbf{u}^{\prime \prime}\right)$.

Conversely, let $\left(\mathbf{u}^{\prime}, \mathbf{u}^{\prime \prime}\right)$ be a saddle point of (2.17) satisfying some boundary condition $\mathbf{u}^{\prime}=\mathbf{u}_{0}^{\prime}$ and $\mathbf{u}^{\prime \prime}=\mathbf{u}_{0}^{\prime \prime}$ on $\partial \Gamma$. Consider

$$
\inf _{\mathbf{s}} \sup _{\mathbf{t}} Q\left(\mathbf{u}^{\prime}+\mathbf{s}, \mathbf{u}^{\prime \prime}+\mathbf{t}\right)
$$

where the infimum and supremum are taken over fields $\mathbf{s}$ and $\mathbf{t}$ satisfying $\mathbf{s}=\mathbf{t}=0$ on $\partial \Gamma$. A necessary condition for the saddle point to be at $\mathbf{s}=\mathbf{t}=0$ is that the first-order derivative of the functional $Q$ vanishes: that is

$$
\int_{\Gamma} 2\left(\begin{array}{c}
\sqcap \mathbf{s} \\
\sqcap \mathbf{t}
\end{array}\right) \cdot\left(\begin{array}{l}
\mathcal{G}^{\prime \prime} \\
\mathcal{G}^{\prime}
\end{array}\right)+2 \mathbf{h}^{\prime \prime} \cdot \mathbf{s}+2 \mathbf{h}^{\prime} \cdot \mathbf{t}=0
$$

for any $\mathbf{s}$ and $\mathbf{t}$ satisfying $\mathbf{s}=\mathbf{t}=0$ on $\partial \Gamma$, where we have introduced

$$
\left(\begin{array}{c}
\mathcal{G}^{\prime \prime} \\
\mathcal{G}^{\prime}
\end{array}\right) \equiv\left(\begin{array}{cc}
\mathbf{Z}^{\prime \prime} & \mathbf{Z}^{\prime} \\
\mathbf{Z}^{\prime} & -\mathbf{Z}^{\prime \prime}
\end{array}\right) \cdot\left(\begin{array}{c}
\sqcap \mathbf{u}^{\prime} \\
\sqcap \mathbf{u}^{\prime \prime}
\end{array}\right)
$$

Upon setting

$$
\mathcal{G}^{\prime}=\left(\begin{array}{c}
\mathrm{G}^{\prime} \\
\mathrm{g}^{\prime}
\end{array}\right), \quad \mathcal{G}^{\prime \prime}=\left(\begin{array}{l}
\mathrm{G}^{\prime \prime} \\
\mathrm{g}^{\prime \prime}
\end{array}\right)
$$

the condition (2.24) reduces to

$$
\int_{\Gamma} \mathbf{G}^{\prime \prime} \cdot \nabla \mathbf{s}+\left(\mathbf{g}^{\prime \prime}+\mathbf{h}^{\prime \prime}\right) \cdot \mathbf{s}+\mathbf{G}^{\prime} \cdot \nabla \mathbf{t}+\left(\mathbf{g}^{\prime}+\mathbf{h}^{\prime}\right) \cdot \mathbf{t}=0 .
$$

Integrating this by parts gives

$$
\int_{\Gamma}\left(-\nabla \cdot \mathbf{G}^{\prime \prime}+\mathbf{g}^{\prime \prime}+\mathbf{h}^{\prime \prime}\right) \cdot \mathbf{s}+\left(-\nabla \cdot \mathbf{G}^{\prime}+\mathbf{g}^{\prime}+\mathbf{h}^{\prime}\right) \cdot \mathbf{t}=0
$$

and this will be satisfied for any $\mathbf{s}$ and $\mathbf{t}$ satisfying $\mathbf{s}=\mathbf{t}=0$ on $\partial \Gamma$ if and only if

$$
\mathbf{h}^{\prime}+\sqcup \mathcal{G}^{\prime}=0, \quad \mathbf{h}^{\prime \prime}+\sqcup \mathcal{G}^{\prime \prime}=0
$$


Thus the Euler-Lagrange equations associated with the saddle point variational principle coincide with the original equations (2.2).

There is also a dual saddle point variational principle, analogous to the Thompson variational principle. By taking real and imaginary parts of the constitutive relation $\mathcal{F}=\mathbf{K} \mathcal{G}$ where $\mathbf{K}=\mathbf{Z}^{-1}$ has negative definite imaginary part we obtain

$$
\left(\begin{array}{c}
\mathcal{F}^{\prime \prime} \\
\mathcal{F}^{\prime}
\end{array}\right)=\left(\begin{array}{cc}
\mathbf{K}^{\prime \prime} & \mathbf{K}^{\prime} \\
\mathbf{K}^{\prime} & -\mathbf{K}^{\prime \prime}
\end{array}\right)\left(\begin{array}{c}
\mathcal{G}^{\prime} \\
\mathcal{G}^{\prime \prime}
\end{array}\right)
$$

The dual variational principle involves the quadratic form

$$
R\left(\underline{\mathbf{G}}^{\prime}, \underline{\mathbf{G}}^{\prime \prime}\right)=\int_{\Gamma}\left(\begin{array}{c}
\underline{\mathcal{G}}^{\prime} \\
\underline{\mathcal{G}}^{\prime \prime}
\end{array}\right) \cdot\left(\begin{array}{cc}
\mathbf{K}^{\prime \prime} & \mathbf{K}^{\prime} \\
\mathbf{K}^{\prime} & -\mathbf{K}^{\prime \prime}
\end{array}\right)\left(\begin{array}{l}
\underline{\mathcal{G}}^{\prime} \\
\underline{\mathcal{G}}^{\prime \prime}
\end{array}\right)=\int_{\Gamma}(\underline{\mathcal{G}} \cdot \mathbf{K} \underline{\mathcal{G}})^{\prime \prime},
$$

where $\underline{\mathcal{G}}^{\prime}$ and $\underline{\mathcal{G}}^{\prime \prime}$ are given by

$$
\underline{\mathcal{G}}^{\prime}=\left(\nabla \cdot \underline{\underline{\mathrm{G}}^{\prime}}-\underline{\mathbf{h}}^{\prime}\right), \quad \underline{\mathcal{G}}^{\prime \prime}=\left(\begin{array}{c}
\underline{\mathrm{G}}^{\prime \prime} \\
\nabla \cdot \underline{\mathrm{G}}^{\prime \prime}-\mathbf{h}^{\prime \prime}
\end{array}\right),
$$

and $\underline{\mathbf{G}}^{\prime}$ and $\underline{\mathbf{G}}^{\prime \prime}$ satisfy the boundary conditions

$$
\underline{\mathbf{G}}^{\prime} \cdot \mathbf{n}=\mathbf{G}^{\prime} \cdot \mathbf{n} \quad \text { and } \underline{\mathbf{G}}^{\prime \prime} \cdot \mathbf{n}=\mathbf{G}^{\prime \prime} \cdot \mathbf{n} \quad \text { on } \partial \Gamma,
$$

where $\mathbf{G}^{\prime}$ and $\mathbf{G}^{\prime \prime}$ are the real and imaginary parts of $\mathbf{G}$. Notice that the form of $\underline{\mathcal{G}}$ given by (2.32) ensures that $\mathbf{h}+\sqcup \underline{\mathcal{G}}=0$. inside $\Gamma$. By similar argument to the one establishing (2.19), we have the dual variational principle

$$
R\left(\mathbf{G}^{\prime}, \mathbf{G}^{\prime \prime}\right)=\sup _{\underline{\mathbf{G}}^{\prime}} \inf _{\mathbf{G}^{\prime \prime}} R\left(\underline{\mathbf{G}}^{\prime}, \underline{\mathbf{G}}^{\prime \prime}\right)=\inf _{\underline{\mathbf{G}}^{\prime \prime}} \sup _{\underline{\mathbf{G}}^{\prime}} R\left(\underline{\mathbf{G}}^{\prime}, \underline{\mathbf{G}^{\prime \prime}}\right),
$$

where the supremum and infimum are over fields satisfying (2.33).

Again building upon the ideas of Cherkaev and Gibiansky (1994) let us rewrite the constitutive relation in the form

$$
\left(\begin{array}{l}
\mathcal{G}^{\prime \prime} \\
\mathcal{F}^{\prime \prime}
\end{array}\right)=\mathcal{L}\left(\begin{array}{c}
\mathcal{F}^{\prime} \\
-\mathcal{G}^{\prime}
\end{array}\right)
$$

where straightforward algebra shows that

$$
\mathcal{L}=\left(\begin{array}{cc}
\mathbf{Z}^{\prime \prime}+\mathbf{Z}^{\prime}\left(\mathbf{Z}^{\prime \prime}\right)^{-1} \mathbf{Z}^{\prime} & \mathbf{Z}^{\prime}\left(\mathbf{Z}^{\prime \prime}\right)^{-1} \\
\left(\mathbf{Z}^{\prime \prime}\right)^{-1} \mathbf{Z}^{\prime} & \left(\mathbf{Z}^{\prime \prime}\right)^{-1}
\end{array}\right)
$$

For all $\mathbf{x} \in \Gamma$ the matrix $\mathcal{L}(\mathbf{x})$ is positive definite since, using (2.14) and (2.35), we see that the associated quadratic form

$$
\begin{aligned}
\left(\begin{array}{c}
\mathcal{F}^{\prime} \\
-\mathcal{G}^{\prime}
\end{array}\right) \cdot \mathcal{L}\left(\begin{array}{c}
\mathcal{F}^{\prime} \\
-\mathcal{G}^{\prime}
\end{array}\right) & =\mathcal{F}^{\prime} \cdot \mathcal{G}^{\prime \prime}-\mathcal{F}^{\prime \prime} \cdot \mathcal{G}^{\prime} \\
& =\mathcal{F}^{\prime} \cdot \mathbf{Z}^{\prime \prime} \mathcal{F}^{\prime}+\mathcal{F}^{\prime \prime} \cdot \mathbf{Z}^{\prime \prime} \mathcal{F}^{\prime \prime}
\end{aligned}
$$


is non-negative, and zero only when $\mathcal{F}^{\prime}=\mathcal{F}^{\prime \prime}=0$, i.e. when $\mathcal{F}^{\prime}=\mathcal{G}^{\prime}=0$.

Now consider the convex functional

$$
Y\left(\underline{\mathbf{u}}^{\prime}, \underline{\mathbf{G}}^{\prime}\right)=\int_{\Gamma}\left(\begin{array}{c}
\sqcap \underline{\mathbf{u}}^{\prime} \\
-\underline{\mathcal{G}}^{\prime}
\end{array}\right) \cdot \mathcal{L}\left(\begin{array}{c}
\sqcap \underline{\mathbf{u}}^{\prime} \\
-\underline{\mathcal{G}}^{\prime}
\end{array}\right)+2 \mathbf{h}^{\prime \prime} \cdot \underline{\mathbf{u}}^{\prime},
$$

where $\underline{\mathcal{G}}^{\prime}$ is given by

$$
\underline{\mathcal{G}}^{\prime}=\left(\nabla \cdot \underline{\mathbf{G}}^{\prime}-\mathbf{h}^{\prime}\right),
$$

(to ensure that $\mathbf{h}^{\prime}+\sqcup \underline{\mathcal{G}}^{\prime}=0$ inside $\Gamma$ ) and $\underline{\mathbf{u}}^{\prime}$ and $\underline{\mathbf{G}}^{\prime}$ are real and satisfy the boundary conditions that

$$
\underline{\mathbf{u}}^{\prime}=\mathbf{u}_{0}^{\prime}, \quad \text { and } \underline{\mathbf{G}}^{\prime} \cdot \mathbf{n}=\mathbf{G}_{0}^{\prime} \cdot \mathbf{n} \quad \text { on } \partial \Gamma .
$$

Let us prove we have the variational principle

$$
Y\left(\mathbf{u}^{\prime}, \mathbf{G}^{\prime}\right)=\inf _{\underline{\mathbf{u}}^{\prime}} \inf _{\underline{\mathbf{G}}^{\prime}} Y\left(\underline{\mathbf{u}}^{\prime}, \underline{\mathbf{G}}^{\prime}\right),
$$

where the infimums are over all trial fields $\underline{\mathbf{u}}^{\prime}$ and $\underline{\mathbf{G}}^{\prime}$ satisfying the boundary conditions (2.40). Let $(\mathbf{u}, \mathcal{G})$ be a solution of (2.2) and (2.7) with $\mathbf{u}^{\prime}=\mathbf{u}_{0}^{\prime}$ and $\mathbf{G}^{\prime} \cdot \mathbf{n}=\mathbf{G}_{0}^{\prime} \cdot \mathbf{n}$ on $\partial \Gamma$. Suppose we are given any $m$ component vector field $\mathbf{s}(\mathbf{x})$ with $\mathbf{s}=0$ on $\partial \Gamma$ and also a $m \times d$ matrix valued field $\mathbf{T}(\mathbf{x})$ with $\mathbf{T} \cdot \mathbf{n}=0$ on $\partial \Gamma$. Then (2.20) and integration by parts implies that

$$
\int_{\Gamma} 2 \mathcal{G}^{\prime \prime} \cdot(\sqcap \mathbf{s})+2 \mathbf{h}^{\prime \prime} \cdot \mathbf{s}-2 \mathcal{T} \cdot\left(\sqcap \mathbf{u}^{\prime \prime}\right)=0, \quad \text { where } \mathcal{T}=\left(\begin{array}{c}
\mathbf{T} \\
\nabla \cdot \mathbf{T}
\end{array}\right) .
$$

From this identity it follows that

$$
Y\left(\mathbf{u}^{\prime}+\mathbf{s}, \mathbf{G}^{\prime}+\mathbf{T}\right)=Y\left(\mathbf{u}^{\prime}, \mathbf{G}^{\prime}\right)+\int_{\Gamma}\left(\begin{array}{c}
\sqcap \mathbf{s} \\
-\mathcal{T}
\end{array}\right) \cdot \mathcal{L}\left(\begin{array}{c}
\sqcap \mathbf{s} \\
-\mathcal{T}
\end{array}\right),
$$

which implies $\left(\mathbf{u}^{\prime}, \mathbf{G}^{\prime}\right)$ is a mimimizer of the variational principle.

Conversely, let $\left(\mathbf{u}^{\prime}, \mathbf{G}^{\prime}\right)$ be a mimimizer of the variational principle, satisfying some boundary conditions $\mathbf{u}^{\prime}=\mathbf{u}_{0}^{\prime}$ and $\mathbf{G}^{\prime} \cdot \mathbf{n}=\mathbf{G}_{0}^{\prime} \cdot \mathbf{n}$ on $\partial \Gamma$. Consider

$$
\inf _{\mathbf{s}} \inf _{\mathbf{T}} Y\left(\mathbf{u}^{\prime}+\mathbf{s}, \mathbf{G}^{\prime}+\mathbf{T}\right)
$$

where the infimum is over all fields $\mathbf{s}$ and $\mathbf{T}$ satisfying $\mathbf{s}=0$ and $\mathbf{T} \cdot \mathbf{n}=0$ on $\partial \Gamma . A$ necessary condition for the infimum to be at $\mathbf{s}=\mathbf{T}=0$ is that the first-order derivative of the functional $Y$ vanish: that is

$$
\int_{\Gamma} 2\left(\begin{array}{c}
\sqcap \mathbf{s} \\
-\mathcal{T}
\end{array}\right) \cdot\left(\begin{array}{c}
\mathcal{G}^{\prime \prime} \\
\mathcal{F}^{\prime \prime}
\end{array}\right)+2 \mathbf{h}^{\prime \prime} \cdot \mathbf{s}=0,
$$

for any $\mathbf{s}$ and $\mathbf{T}$ satisfying $\mathbf{s}=0$ and $\mathbf{T} \cdot \mathbf{n}=0$ on $\partial \Gamma$, where we have introduced

$$
\mathcal{T} \equiv\left(\begin{array}{c}
\mathbf{T} \\
\nabla \cdot \mathbf{T}
\end{array}\right), \quad\left(\begin{array}{c}
\mathcal{G}^{\prime \prime} \\
\mathcal{F}^{\prime \prime}
\end{array}\right) \equiv \mathcal{L}\left(\begin{array}{c}
\sqcap \mathbf{u}^{\prime} \\
-\mathcal{G}^{\prime}
\end{array}\right)
$$


Upon setting

$$
\mathcal{F}^{\prime \prime}=\left(\begin{array}{c}
\mathbf{F}^{\prime \prime} \\
\mathbf{f}^{\prime \prime}
\end{array}\right), \quad \mathcal{G}^{\prime \prime}=\left(\begin{array}{c}
\mathrm{G}^{\prime \prime} \\
\mathbf{g}^{\prime \prime}
\end{array}\right),
$$

the condition (2.45) reduces to

$$
\int_{\Gamma} \mathrm{G}^{\prime \prime} \cdot \nabla \mathbf{s}+\left(\mathrm{g}^{\prime \prime}+\mathbf{h}^{\prime \prime}\right) \cdot \mathbf{s}-\mathbf{T} \cdot \mathbf{F}^{\prime \prime}-(\nabla \cdot \mathbf{T}) \cdot \mathbf{f}^{\prime \prime}=0 .
$$

Integrating this by parts gives

$$
\int_{\Gamma}\left(-\nabla \cdot \mathrm{G}^{\prime \prime}+\mathrm{g}^{\prime \prime}+\mathbf{h}^{\prime \prime}\right) \cdot \mathbf{s}-\mathbf{T} \cdot\left(\mathbf{F}^{\prime \prime}-\nabla \mathbf{f}^{\prime \prime}\right)=0,
$$

and this will be satisfied for any $\mathbf{s}$ and $\mathbf{T}$ satisfying $\mathbf{s}=0$ and $\mathbf{T} \cdot \mathbf{n}=0$ on $\partial \Gamma$ if and only if

$$
\mathbf{h}^{\prime \prime}+\sqcup \mathcal{G}^{\prime \prime}=0, \quad \mathcal{F}^{\prime \prime}=\sqcap \mathbf{f}^{\prime \prime} .
$$

Upon identifying $\mathbf{f}^{\prime \prime}$ with $\mathbf{u}^{\prime \prime}$ we see that the Euler-Lagrange equations associated with the minimization variational principle coincide with the original equations (2.2).

The convex variational principle we have just stated is, as usual, associated with a dual variational principle:

$$
\widetilde{Y}\left(\mathbf{u}^{\prime \prime}, \mathbf{G}^{\prime \prime}\right)=\inf _{\underline{\mathbf{u}}^{\prime \prime}} \inf _{\mathbf{G}^{\prime \prime}} \widetilde{Y}\left(\underline{\mathbf{u}}^{\prime \prime}, \underline{\mathbf{G}}^{\prime \prime}\right),
$$

where

$$
\tilde{Y}\left(\underline{\mathbf{u}}^{\prime \prime}, \underline{\mathbf{G}}^{\prime \prime}\right)=\int_{\Gamma}\left(\frac{\underline{\mathcal{G}}^{\prime \prime}}{\sqcap \underline{\mathbf{u}}^{\prime \prime}}\right) \cdot \mathcal{L}^{-1}\left(\frac{\mathcal{G}^{\prime \prime}}{\sqcap \underline{\mathbf{u}}^{\prime \prime}}\right)-2 \mathbf{h}^{\prime} \cdot \underline{\mathbf{u}}^{\prime \prime},
$$

in which

$$
\underline{\mathcal{G}}^{\prime \prime}=\left(\nabla \cdot \underline{\mathrm{G}}^{\prime \prime}-\underline{\mathbf{G}}^{\prime \prime}\right),
$$

and the infimum in (2.51) is over real fields $\underline{\mathbf{u}}^{\prime \prime}$ and $\underline{\mathbf{G}}^{\prime \prime}$ with $\underline{\mathbf{u}}^{\prime \prime}=\mathbf{u}^{\prime \prime}$ and $\underline{\mathbf{G}}^{\prime \prime} \cdot \mathbf{n}=\mathbf{G}^{\prime \prime} \cdot \mathbf{n}$ on $\partial \Gamma$. Since

$$
\mathcal{L}^{-1}=\left(\begin{array}{cc}
\left(\mathbf{Z}^{\prime \prime}\right)^{-1} & -\left(\mathbf{Z}^{\prime \prime}\right)^{-1} \mathbf{Z}^{\prime} \\
-\mathbf{Z}^{\prime}\left(\mathbf{Z}^{\prime \prime}\right)^{-1} & \mathbf{Z}^{\prime \prime}+\mathbf{Z}^{\prime}\left(\mathbf{Z}^{\prime \prime}\right)^{-1} \mathbf{Z}^{\prime}
\end{array}\right),
$$

the expression for $\widetilde{Y}\left(\underline{\mathbf{u}}^{\prime \prime}, \underline{\mathrm{G}}^{\prime \prime}\right)$ can be written equivalently as

$$
\widetilde{Y}\left(\underline{\mathbf{u}}^{\prime \prime}, \underline{\mathbf{G}}^{\prime \prime}\right)=\int_{\Gamma}\left(\begin{array}{l}
\sqcap \underline{\mathbf{u}}^{\prime \prime} \\
-\underline{\mathcal{G}}^{\prime \prime}
\end{array}\right) \cdot \mathcal{L}\left(\begin{array}{l}
\sqcap \underline{\mathbf{u}}^{\prime \prime} \\
-\underline{\mathcal{G}}^{\prime \prime}
\end{array}\right)-2 \mathbf{h}^{\prime} \cdot \underline{\mathbf{u}}^{\prime \prime} .
$$

These two minimization variational principles are not the only ones one can derive. Indeed, there is a continuous two-parameter family of related variational principles. The differential constraints (2.2) and the constitutive relation (2.7) imply

$$
\widetilde{\mathcal{F}}=\sqcap \widetilde{\mathbf{u}}, \quad \widetilde{\mathbf{h}}+\sqcup \widetilde{\mathcal{G}}=0, \quad \widetilde{\mathcal{G}}(\mathbf{x})=\widetilde{\mathbf{Z}}(\mathbf{x}) \widetilde{\mathcal{F}}(\mathbf{x}),
$$


where

$$
\widetilde{\mathbf{u}}=e^{i \tau} \mathbf{u}, \quad \widetilde{\mathcal{F}}=e^{i \tau} \mathcal{F}, \quad \widetilde{\mathcal{G}}=e^{i(\tau+\theta)} \mathcal{G}, \quad \widetilde{\mathbf{h}}=e^{i(\tau+\theta)} \mathbf{h}, \quad \widetilde{\mathbf{Z}}(\mathbf{x})=e^{i \theta} \mathbf{Z}(\mathbf{x}),
$$

in which $\tau$ and $\theta$ are real parameters. So the fields with tildes on them satisfy the same equations as those without tildes, and the variational principles directly apply provided $\theta$ is chosen so that

$$
\widetilde{\mathbf{Z}}^{\prime \prime}(\mathbf{x}) \geq \alpha \mathbf{I}, \quad \forall \mathbf{x} \in \Gamma,
$$

for some $\alpha>0$. With the subsequent replacement (2.57) we obtain a new set of variational principles parameterized by $\tau$ and $\theta$. Observe that even if (2.15) is not satisfied for any $\alpha>0$ we still may be able to find a range of values of $\theta$ for which (2.58) holds, and thus for which we can obtain variational principles. With the particular choice $\theta=0$ and $e^{i \tau}=-i$ the variational principle (2.41) gets mapped to the dual variational principle (2.51).

All the previous minimization principles need the fundamental coercivity assumption (2.15). If $\mathbf{Z}^{\prime \prime}(\mathbf{x})=0$ in some part $\Psi$ of the domain $\Gamma$ they cannot be applied directly. However we can still obtain variational principles by taking appropriate limits. If, in some region $\Psi \subset \Gamma, \mathbf{Z}^{\prime \prime}(\mathbf{x})$ is very small in the sense that

$$
\delta \mathbf{I} \geq \mathbf{Z}^{\prime \prime}(\mathbf{x}), \quad \forall \mathbf{x} \in \Psi,
$$

for some very small $\delta>0$, then within this region

$$
\left(\begin{array}{c}
\sqcap \underline{\mathbf{u}}^{\prime} \\
-\underline{\mathcal{G}}^{\prime}
\end{array}\right) \cdot \mathcal{L}\left(\begin{array}{c}
\sqcap \underline{\mathbf{u}}^{\prime} \\
-\underline{\mathcal{G}}^{\prime}
\end{array}\right) \approx\left[\mathbf{Z}^{\prime} \sqcap \underline{\mathbf{u}}^{\prime}-\underline{\mathcal{G}}^{\prime}\right] \cdot\left(\mathbf{Z}^{\prime \prime}\right)^{-1}\left[\mathbf{Z}^{\prime} \sqcap \underline{\mathbf{u}}^{\prime}-\underline{\mathcal{G}}^{\prime}\right] .
$$

If $\Psi=\Gamma$ then the variational principle reduces to a variational principle similar to (1.12) which is not particularly interesting. If $\Psi \neq \Gamma$ the variational principle will only be useful if one takes $\underline{\mathcal{G}}^{\prime} \approx \mathbf{Z}^{\prime} \sqcap \underline{\mathbf{u}}^{\prime}$ in $\Psi$. The variational principles still hold in the limit as $\delta \rightarrow 0$ provided one first takes

$$
\underline{\mathcal{G}}^{\prime}=\mathbf{Z}^{\prime} \sqcap \underline{\mathbf{u}}^{\prime}, \quad \forall \mathbf{x} \in \Psi,
$$

corresponding to taking an exact solution of (2.11) in this region (but with $\left(\underline{\mathbf{u}}^{\prime}, \underline{\mathbf{G}}^{\prime}\right)$ not necessarily coinciding with the exact solution $\left(\mathbf{u}^{\prime}, \mathbf{G}^{\prime}\right)$ associated with the given boundary conditions). Then as $\delta \rightarrow 0$ the expression for $Y$ reduces to

$$
\widetilde{Y}\left(\underline{\mathbf{u}}^{\prime}, \underline{\mathbf{G}}^{\prime}\right)=\int_{\Gamma \backslash \Psi}\left(\begin{array}{l}
\sqcap \underline{\mathbf{u}}^{\prime} \\
-\underline{\mathcal{G}^{\prime}}
\end{array}\right) \cdot \mathcal{L}\left(\begin{array}{l}
\sqcap \underline{\mathbf{u}}^{\prime} \\
-\underline{\mathcal{G}}^{\prime}
\end{array}\right)-\int_{\Gamma} 2 \mathbf{h}^{\prime \prime} \cdot \underline{\mathbf{u}}^{\prime} .
$$

Outside $\Psi$ we still require that $\mathbf{Z}^{\prime \prime}(\mathbf{x}) \geq \alpha \mathbf{I}$ for some $\alpha>0$.

We now show how these variational principles may give useful information about the Dirichlet to Neumann map on the boundary $\partial \Gamma$. Let us assume $\mathbf{h}=0$ and introduce the $m$-component complex vector fields

$$
\mathbf{q}_{0}=\mathbf{G}(\mathbf{x}) \cdot \mathbf{n}, \quad \mathbf{u}_{0}(\mathbf{x})=\mathbf{u}(\mathbf{x}) \quad \text { for } \mathbf{x} \in \partial \Gamma .
$$


The relation between $\mathbf{q}_{0}$ and $\mathbf{u}_{0}$ must be linear and we can write $\mathbf{q}_{0}=\mathbf{N} \mathbf{u}_{0}$ which defines the Dirichlet to Neumann map $\mathbf{N}$. If $\mathbf{u}_{0}^{(1)}$ and $\mathbf{u}_{0}^{(2)}$ denote two boundary conditions for $\mathbf{u}$ and $\mathcal{G}^{(j)}, \mathcal{F}^{(j)}$, and $\mathbf{q}_{0}^{(j)}, j=1,2$, denote the associated fields then it follows from the key property and the symmetry of $\mathbf{Z}(\mathbf{x})$ that

$$
\begin{aligned}
\left\langle\mathbf{N u}_{0}^{(1)}, \mathbf{u}_{0}^{(2)}\right\rangle & =\int_{\partial \Gamma} \mathbf{q}_{0}^{(1)} \cdot \mathbf{u}_{0}^{(2)}=\int_{\Gamma} \mathcal{G}^{(1)} \cdot \mathcal{F}^{(2)}=\int_{\Gamma} \mathcal{F}^{(1)} \cdot \mathbf{Z} \mathcal{F}^{(2)} \\
& =\int_{\Gamma} \mathcal{G}^{(2)} \cdot \mathcal{F}^{(1)}=\int_{\partial \Gamma} \mathbf{q}_{0}^{(2)} \cdot \mathbf{u}_{0}^{(1)}=\left\langle\mathbf{u}_{0}^{(1)}, \mathbf{N} \mathbf{u}_{0}^{(2)}\right\rangle,
\end{aligned}
$$

which implies the map $\mathbf{N}$ is symmetric. It has positive semidefinite imaginary part since

$$
\begin{aligned}
\left\langle\mathbf{u}_{0}^{\prime}, \mathbf{N}^{\prime \prime} \mathbf{u}_{0}^{\prime}\right\rangle & +\left\langle\mathbf{u}_{0}^{\prime \prime}, \mathbf{N}^{\prime \prime} \mathbf{u}_{0}^{\prime \prime}\right\rangle=\int_{\partial \Gamma} \mathbf{q}_{0}^{\prime \prime} \cdot \mathbf{u}_{0}^{\prime}-\mathbf{q}_{0}^{\prime} \cdot \mathbf{u}_{0}^{\prime \prime} \\
& =\int_{\Gamma} \mathcal{G}^{\prime \prime} \cdot \mathcal{F}^{\prime}-\mathcal{G}^{\prime} \cdot \mathcal{F}^{\prime \prime}=\int_{\Gamma} \mathcal{F}^{\prime} \cdot \mathbf{Z}^{\prime \prime} \mathcal{F}^{\prime}+\mathcal{F}^{\prime \prime} \cdot \mathbf{Z}^{\prime \prime} \mathcal{F}^{\prime \prime} \geq 0 .
\end{aligned}
$$

Assuming $\mathbf{N}^{\prime \prime}$ is invertible, we can write the relation $\mathbf{q}_{0}=\mathbf{N} \mathbf{u}_{0}$ as

$$
\left(\begin{array}{c}
\mathbf{q}_{0}^{\prime \prime} \\
\mathbf{u}_{0}^{\prime \prime}
\end{array}\right)=\mathcal{N}\left(\begin{array}{c}
\mathbf{u}_{0}^{\prime} \\
-\mathbf{q}_{0}^{\prime}
\end{array}\right)
$$

where

$$
\mathcal{N}=\left(\begin{array}{cc}
\mathbf{N}^{\prime \prime}+\mathbf{N}^{\prime}\left(\mathbf{N}^{\prime \prime}\right)^{-1} \mathbf{N}^{\prime} & \mathbf{N}^{\prime}\left(\mathbf{N}^{\prime \prime}\right)^{-1} \\
\left(\mathbf{N}^{\prime \prime}\right)^{-1} \mathbf{N}^{\prime} & \left(\mathbf{N}^{\prime \prime}\right)^{-1}
\end{array}\right)
$$

Thus the map $\mathcal{N}$ giving the boundary fields $\mathbf{q}_{0}^{\prime \prime}$ and $\mathbf{u}_{0}^{\prime \prime}$ in terms of $\mathbf{q}_{0}^{\prime}$ and $\mathbf{u}_{0}^{\prime}$ must have this special form.

Now, using the key property, $Y\left(\mathbf{u}^{\prime}, \mathbf{G}^{\prime}\right)$ can be expressed (when $\mathbf{h}=0$ ) in terms of the boundary fields:

$$
\begin{aligned}
Y\left(\mathbf{u}^{\prime}, \mathbf{G}^{\prime}\right) & =\int_{\Gamma} \mathcal{G}^{\prime \prime} \cdot \mathcal{F}^{\prime}-\mathcal{G}^{\prime} \cdot \mathcal{F}^{\prime \prime}=\int_{\partial \Gamma} \mathbf{q}_{0}^{\prime \prime} \cdot \mathbf{u}_{0}^{\prime}-\mathbf{q}_{0}^{\prime} \cdot \mathbf{u}_{0}^{\prime \prime} \\
& =\left\langle\left(\begin{array}{c}
\mathbf{u}_{0}^{\prime} \\
-\mathbf{q}_{0}^{\prime}
\end{array}\right), \mathcal{N}\left(\begin{array}{c}
\mathbf{u}_{0}^{\prime} \\
-\mathbf{q}_{0}^{\prime}
\end{array}\right)\right\rangle
\end{aligned}
$$

and thus bounds on $Y\left(\mathbf{u}^{\prime}, \mathbf{G}^{\prime}\right)$, obtained from the variational principle, give upper bounds on the quadratic form associated with $\mathcal{N}$. Bounds on $\widetilde{Y}\left(\mathbf{u}^{\prime \prime}, \mathbf{G}^{\prime \prime}\right)$, obtained from the dual variational principle, give lower bounds on the quadratic form associated with $\mathcal{N}$.

\section{Application to Acoustics and Elastodynamics}

Let us begin by studying the acoustic equation in $d$ dimensions:

$$
-\nabla \cdot \boldsymbol{\rho}^{-1} \nabla P=(1 / \kappa) \omega^{2} P
$$


where $P(\mathbf{x})$ is the complex pressure, $\kappa(\mathbf{x})$ is the bulk modulus, $\boldsymbol{\rho}(\mathbf{x})$ is the density tensor, and $\omega$ is the (fixed) frequency of oscillation. [As is well known this equation with $d=2$ is also applicable in cylindrical bodies, for antiplane elastodynamics where the displacement field is directed parallel to the cylinder axis, and for electromagnetism when the fields are transverse electric (TE), where the electric field is directed parallel to the cylinder axis, or transverse magnetic (TM), where the magnetic field is directed parallel to the cylinder axis]. Normally one expects $\kappa$ to have a negative imaginary part (due to bulk viscosity), and $\boldsymbol{\rho}$ to be $\rho \mathbf{I}$ where $\rho(\mathbf{x})$ is the positive mass density. However, viewed as the effective density tensor of a composite material at a given frequency $\boldsymbol{\omega}, \boldsymbol{\rho}$ can be anisotropic (Schoenberg and Sen 1983) and, at least in the context of elastodynamics, can be complex valued with a positive semidefinite imaginary part (Milton and Willis 2007) with a real part that is not even necessarily positive [as established by Movchan and Guenneau (2004), Bouchitté and Felbacq (2004), and Felbacq and Bouchitté (2005) in the context of antiplane elastodynamics, where $1 / \kappa$ plays the role of density, and by Liu, Chan, and Sheng (2005) and Ávila, Griso, and Miara (2005) in the context of three-dimensional elastodynamics]. Such unusual effective densities are due to the fact that different parts of the microstructure can experience different accelerations, and can be out of phase with the overall applied force if this relative motion dissipates energy.

Given the complex pressure field $P(\mathbf{x})$ the associated complex velocity field of the fluid is

$$
\mathbf{v}=-i(\omega \boldsymbol{\rho})^{-1} \nabla P
$$

[The associated physical pressure and physical velocity are respectively $\left(e^{-i \omega t} P(\mathbf{x})\right)^{\prime}$ and $\left(e^{-i \omega t} \mathbf{v}(\mathbf{x})\right)^{\prime}$ where the prime denotes the real part and $t$ denotes the time.] Upon multiplying (3.1) by $e^{i \theta}$ and comparing it with (2.12) we can make the identifications

$$
\mathbf{u}=P, \quad \mathbf{L}=-e^{i \theta} \boldsymbol{\rho}^{-1}, \quad \mathbf{K}=0, \quad \mathbf{M}=e^{i \theta} \omega^{2} / \kappa, \quad \mathbf{h}=0, \quad \mathbf{G}=-i e^{i \theta} \omega \mathbf{v} .
$$

Thus the potential $\mathbf{u}$ is a scalar, corresponding to the case $m=1$. We choose $\theta$ such that the imaginary part of

$$
\mathbf{Z}=\left(\begin{array}{cc}
-e^{i \theta} \boldsymbol{\rho}^{-1} & 0 \\
0 & e^{i \theta} \omega^{2} / \kappa
\end{array}\right)
$$

satisfies (2.15). When $\boldsymbol{\rho}$ is real and positive definite, as is typically the case, then we need to choose $\theta$ in the range $0>\theta>-\pi$. If in addition $\kappa$ is almost real and positive, with a small negative imaginary part, then we should choose $\theta$ appropriately small and negative.

Explicit expressions for the various variational principles in the acoustic case can of course be obtained by making the substitutions (3.3) in the relevant equations in the preceding section. For example, if $\boldsymbol{\rho}$ has a positive definite imaginary part (which is more applicable in the mathematically analogous TE or TM electromagnetic problems) and $\kappa(\mathbf{x})$ has a negative imaginary part, then with $\theta=0$ we have the variational principle

$$
Y\left(P^{\prime}, \mathbf{v}^{\prime \prime}\right)=\inf _{\underline{P}^{\prime}} \inf _{\mathbf{v}^{\prime \prime}} Y\left(\underline{P}^{\prime}, \underline{\mathbf{v}}^{\prime \prime}\right)
$$


where

$$
Y\left(\underline{P}^{\prime}, \underline{\mathbf{v}}^{\prime \prime}\right)=\int_{\Gamma}\left(\begin{array}{c}
\nabla \underline{P}^{\prime} \\
-\omega \underline{\mathbf{v}}^{\prime \prime}
\end{array}\right) \cdot \mathcal{R}\left(\begin{array}{c}
\nabla \underline{P}^{\prime} \\
-\omega \underline{\mathbf{v}}^{\prime \prime}
\end{array}\right)+\left(\begin{array}{c}
\omega \underline{P}^{\prime} \\
-\nabla \cdot \underline{\mathbf{v}}^{\prime \prime}
\end{array}\right) \cdot \mathcal{K}\left(\begin{array}{c}
\omega \underline{P^{\prime}} \\
-\nabla \cdot \underline{\mathbf{v}}^{\prime \prime}
\end{array}\right),
$$

in which

$$
\begin{aligned}
& \mathcal{R}=\left(\begin{array}{cc}
\mathbf{r}^{\prime \prime}+\mathbf{r}^{\prime}\left(\mathbf{r}^{\prime \prime}\right)^{-1} \mathbf{r}^{\prime} & \mathbf{r}^{\prime}\left(\mathbf{r}^{\prime \prime}\right)^{-1} \\
\left(\mathbf{r}^{\prime \prime}\right)^{-1} \mathbf{r}^{\prime} & \left(\mathbf{r}^{\prime \prime}\right)^{-1}
\end{array}\right), \\
& \mathcal{K}=\left(\begin{array}{cc}
k^{\prime \prime}+\left(k^{\prime}\right)^{2} / k^{\prime \prime} & k^{\prime} / k^{\prime \prime} \\
k^{\prime} / k^{\prime \prime} & 1 / k^{\prime \prime}
\end{array}\right),
\end{aligned}
$$

and $\mathbf{r}=-\boldsymbol{\rho}^{-1}$, and $k=1 / \kappa$. In this variational principle the boundary values of $\underline{P}^{\prime}$ and $\underline{\mathbf{v}}^{\prime \prime} \cdot \mathbf{n}$ are fixed, and the infimums are attained at fields $P^{\prime}$ and $\mathbf{v}^{\prime \prime}$ associated with solutions of the acoustic equation satisfying the prescribed boundary conditions. That the EulerLagrange equations of (3.5) coincide with (3.1) and (3.2) follows from the analysis of (2.44)-(2.50), and can also be shown directly.

When $\mathbf{r}^{\prime \prime}$ is very small within all of $\Gamma$ then we have

$$
\left(\begin{array}{c}
\nabla \underline{P}^{\prime} \\
-\omega \underline{\mathbf{v}}^{\prime \prime}
\end{array}\right) \cdot \mathcal{R}\left(\begin{array}{c}
\nabla \underline{P}^{\prime} \\
-\omega \underline{\mathbf{v}}^{\prime \prime}
\end{array}\right) \approx\left[\mathbf{r}^{\prime} \nabla \underline{P}^{\prime}-\omega \underline{\mathbf{v}}^{\prime \prime}\right] \cdot\left(\mathbf{r}^{\prime \prime}\right)^{-1}\left[\mathbf{r}^{\prime} \nabla \underline{P}^{\prime}-\omega \underline{\mathbf{v}}^{\prime \prime}\right]
$$

and the variational principles will only be useful if we take trial fields with $\omega \underline{\mathbf{v}^{\prime \prime}} \approx \mathbf{r}^{\prime} \nabla \underline{P^{\prime}}$. The variational principles still hold in the limit as $\mathbf{r}^{\prime \prime} \rightarrow 0$ provided we first choose $\underline{\mathbf{v}}^{\prime \prime}=\mathbf{r}^{\prime} \nabla \underline{P}^{\prime} / \omega$. Then, when $\boldsymbol{\rho}$ is real, we have the variational principle

$$
Y\left(P^{\prime}\right)=\inf _{\underline{P}^{\prime}} Y\left(\underline{P}^{\prime}\right)
$$

where

$$
Y\left(\underline{P}^{\prime}\right)=\int_{\Gamma}\left(\begin{array}{c}
\omega \underline{P^{\prime}} \\
\nabla \cdot \rho^{-1} \nabla \underline{P}^{\prime} / \omega
\end{array}\right) \cdot \mathcal{K}\left(\begin{array}{c}
\omega \underline{P}^{\prime} \\
\nabla \cdot \rho^{-1} \nabla \underline{P}^{\prime} / \omega
\end{array}\right) .
$$

In this variational principle the boundary values of $\underline{P}^{\prime}$ and $\mathbf{n} \cdot \boldsymbol{\rho}^{-1} \nabla \underline{P^{\prime}}$ are fixed, where the latter corresponds to fixing the boundary value of $\underline{\mathbf{v}}^{\prime \prime} \cdot \mathbf{n}$. The infimum is attained at a field $P^{\prime}$ associated with solutions of the acoustic equation satisfying the prescribed boundary conditions. To show this, let $P^{\prime}$ be a minimizer and set $\underline{P}^{\prime}=P^{\prime}+S$ where $S=0$ and $\mathbf{n} \cdot \boldsymbol{\rho}^{-1} \nabla S=0$ on $\partial \Gamma$. A necessary condition for the minimum to be at $S=0$ is that the first derivative of the functional $Y$ vanish: that is

$$
\int_{\Gamma}\left(\begin{array}{c}
U \\
\omega P^{\prime \prime}
\end{array}\right) \cdot\left(\begin{array}{c}
\omega S \\
\nabla \cdot \boldsymbol{\rho}^{-1} \nabla S / \omega
\end{array}\right)=0
$$

where we have introduced

$$
\left(\begin{array}{c}
U \\
\omega P^{\prime \prime}
\end{array}\right) \equiv \mathcal{K}\left(\begin{array}{c}
\omega \underline{P^{\prime}} \\
\nabla \cdot \rho^{-1} \nabla \underline{P}^{\prime} / \omega
\end{array}\right)
$$


Integrating by parts the constraint (3.11) gives

$$
\int_{\Gamma} S\left(\omega U+\nabla \cdot \boldsymbol{\rho}^{-1} \nabla \underline{P}^{\prime \prime}\right)=0
$$

and this will be satisfied for any $S$ with $S=0$ and $\mathbf{n} \cdot \boldsymbol{\rho}^{-1} \nabla S=0$ on $\partial \Gamma$ if and only if

$$
U=-\nabla \cdot \rho^{-1} \nabla \underline{P}^{\prime \prime} / \omega
$$

A straightforward calculation (similar to the equivalence of (2.7) and (2.35)) shows that (3.12) is equivalent to (3.1) with this value of $U$. Thus the acoustic equation is the Euler-Lagrange equation of (3.9), when $P^{\prime \prime}$ is defined by (3.12). This variational principle remains valid if we replace $P$ by $e^{i \tau} P$ where $\tau$ can be any constant.

In a similar way, if $\boldsymbol{\rho}$ has a positive definite imaginary part but $\kappa$ is real (which is more applicable to the analogous TM electromagnetic problem, where the magnetic permeability plays the role of $1 / \kappa)$ then we have the variational principle

$$
Y\left(\mathbf{v}^{\prime \prime}\right)=\inf _{\underline{\mathbf{v}}^{\prime \prime}} Y\left(\underline{\mathbf{v}}^{\prime \prime}\right),
$$

where

$$
Y\left(\underline{\mathbf{v}}^{\prime \prime}\right)=\int_{\Gamma}\left(\begin{array}{c}
\nabla\left(\kappa \nabla \cdot \underline{\mathbf{v}}^{\prime \prime}\right) / \omega \\
-\omega \underline{\mathbf{v}}^{\prime \prime}
\end{array}\right) \cdot \mathcal{R}\left(\begin{array}{c}
\nabla\left(\kappa \nabla \cdot \underline{\mathbf{v}}^{\prime \prime}\right) / \omega \\
-\omega \underline{\mathbf{v}}^{\prime \prime}
\end{array}\right),
$$

in which the boundary values of $\underline{\mathbf{v}}^{\prime \prime} \cdot \mathbf{n}$ and $\kappa \nabla \cdot \underline{\mathbf{v}}^{\prime \prime}$ are fixed, where the latter corresponds to fixing the boundary value of $\underline{P}^{\prime}$.

One has to slightly modify the analysis to apply it to the elastodynamic equations,

$$
-\nabla \cdot(\mathbf{C} \nabla \mathbf{u})=\mathbf{b}+\omega^{2} \boldsymbol{\rho} \mathbf{u}
$$

in which $\mathbf{u}(\mathbf{x})$ is the complex displacement field, $\mathbf{b}(\mathbf{x})$ is the complex body-force density $\boldsymbol{\rho}(\mathbf{x})$ is the complex density tensor, and $\mathbf{C}(\mathbf{x})$ is the complex elasticity tensor. Given the complex displacement field $\mathbf{u}(\mathbf{x})$, the complex stress $\boldsymbol{\tau}(\mathbf{x})$ and complex momentum density $p(\mathbf{x})$ are given by

$$
\boldsymbol{\tau}=\mathbf{C} \nabla \mathbf{u}, \quad \mathbf{p}=-i \omega \rho \mathbf{u}
$$

[The associated physical displacement, body-force density, stress, and momentum density are respectively $\left(e^{-i \omega t} \mathbf{u}\right)^{\prime},\left(e^{-i \omega t} \mathbf{b}\right)^{\prime},\left(e^{-i \omega t} \boldsymbol{\tau}\right)^{\prime}$ and $\left(e^{-i \omega t} \mathbf{p}\right)^{\prime}$.] Upon multiplying (3.17) by $e^{i \theta}$ and comparing it with (2.12) we can make the identifications

$$
\mathbf{L}=-e^{i \theta} \mathbf{C}, \quad \mathbf{K}=0, \quad \mathbf{M}=e^{i \theta} \omega^{2} \boldsymbol{\rho}, \quad \mathbf{h}=e^{i \theta} \mathbf{b},
$$

and (3.18) implies

$$
\mathbf{G}=-e^{i \theta} \boldsymbol{\tau}, \quad \mathbf{g}=i e^{i \theta} \omega \mathbf{p} .
$$

The problem is that $\mathbf{C}$ acting upon any antisymmetric matrix is zero, and so $\mathbf{L}$ and hence $\mathbf{Z}$ are singular, which is a problem for computing the inverses in the equation (2.36) for 
$\mathcal{L}$. This problem is rectified in the standard way, by replacing $\sqcap$ everywhere with $\widetilde{\Pi}$ defined by

$$
\widetilde{\sqcap} \mathbf{u} \equiv\left(\begin{array}{c}
{\left[\nabla \mathbf{u}+(\nabla \mathbf{u})^{T}\right] / 2} \\
\mathbf{u}
\end{array}\right) .
$$

Then the fields $\mathcal{F}$ and $\mathcal{G}$ have the form (2.1), with $\mathbf{F}$ and $\mathbf{G}$ being symmetric $d \times d$ matrices: thus $\mathcal{F}$ and $\mathcal{G}$ can be represented by $d(d+3) / 2$ component vectors. We choose $\theta$ such that the imaginary part of

$$
\mathbf{Z}=\left(\begin{array}{cc}
-e^{i \theta} \mathbf{C} & 0 \\
0 & e^{i \theta} \omega^{2} \boldsymbol{\rho}
\end{array}\right)
$$

satisfies (2.15) on the space of these fields, and we compute the inverse of $\mathbf{Z}^{\prime \prime}$ on the space of these fields. When $\boldsymbol{\rho}$ is real and positive definite then we need to choose $\theta$ in the range $\pi>\theta>0$. If in addition $\mathbf{C}$ is almost real with a positive definite real part, and with a small negative definite imaginary part, then we should choose $\theta$ appropriately small and positive. With $\sqcap$ replaced by $\widetilde{\Pi}$ the analysis of equations (2.23) $-(2.29)$ and (2.44)(2.50) still holds, and we recover the elastodynamic equations from the Euler-Lagrange equations associated with the variational principles.

In the special case $\theta=0$ the functionals $Q, R$ and $Y$ reduce to

$$
\begin{aligned}
& Q\left(\underline{\mathbf{u}}^{\prime}, \underline{\mathbf{u}}^{\prime \prime}\right)=\int_{\Gamma}\left(-\underline{\boldsymbol{\epsilon}} \cdot \mathbf{C} \underline{\boldsymbol{\epsilon}}+\omega^{2} \underline{\mathbf{u}} \cdot \boldsymbol{\rho} \underline{\mathbf{u}}+2 \mathbf{b} \cdot \underline{\mathbf{u}}\right)^{\prime \prime}, \\
& R\left(\underline{\boldsymbol{\tau}}^{\prime}, \underline{\boldsymbol{\tau}}^{\prime \prime}\right)= \int_{\Gamma}\left[-\underline{\boldsymbol{\tau}} \cdot \mathbf{C}^{-1} \underline{\boldsymbol{\tau}}+(\nabla \cdot \underline{\boldsymbol{\tau}}+\mathbf{b}) \cdot\left(\omega^{2} \boldsymbol{\rho}\right)^{-1}(\nabla \cdot \underline{\boldsymbol{\tau}}+\mathbf{b})\right]^{\prime \prime}, \\
& Y\left(\left(\underline{\mathbf{u}}^{\prime}, \underline{\boldsymbol{\tau}}^{\prime}\right)\right)=\int_{\Gamma}-\left(\begin{array}{c}
\underline{\boldsymbol{\epsilon}}^{\prime} \\
-\underline{\boldsymbol{\tau}}^{\prime}
\end{array}\right) \cdot \mathcal{C}\left(\begin{array}{c}
\underline{\boldsymbol{\epsilon}}^{\prime} \\
-\underline{\boldsymbol{\tau}}^{\prime}
\end{array}\right) \\
&+\left(\begin{array}{c}
\omega \underline{\mathbf{u}}^{\prime} \\
\left(\mathbf{b}^{\prime}+\nabla \cdot \underline{\boldsymbol{\tau}}^{\prime}\right) / \omega
\end{array}\right) \mathcal{P}\left(\begin{array}{c}
\omega \underline{\mathbf{u}}^{\prime} \\
\left(\mathbf{b}^{\prime}+\nabla \cdot \underline{\boldsymbol{\tau}}^{\prime}\right) / \omega
\end{array}\right)+2 \mathbf{b}^{\prime \prime} \cdot \underline{\mathbf{u}}^{\prime},
\end{aligned}
$$

where

$$
\underline{\boldsymbol{\epsilon}}=\left[\nabla \underline{\mathbf{u}}+(\nabla \underline{\mathbf{u}})^{T}\right] / 2,
$$

and

$$
\begin{aligned}
\mathcal{C} & =\left(\begin{array}{cc}
\mathbf{C}^{\prime \prime}+\mathbf{C}^{\prime}\left(\mathbf{C}^{\prime \prime}\right)^{-1} \mathbf{C}^{\prime} & \mathbf{C}^{\prime}\left(\mathbf{C}^{\prime \prime}\right)^{-1} \\
\left(\mathbf{C}^{\prime \prime}\right)^{-1} \mathbf{C}^{\prime} & \left(\mathbf{C}^{\prime \prime}\right)^{-1}
\end{array}\right), \\
\mathcal{P} & =\left(\begin{array}{cc}
\boldsymbol{\rho}^{\prime \prime}+\boldsymbol{\rho}^{\prime}\left(\boldsymbol{\rho}^{\prime \prime}\right)^{-1} \boldsymbol{\rho}^{\prime} & \boldsymbol{\rho}^{\prime}\left(\boldsymbol{\rho}^{\prime \prime}\right)^{-1} \\
\left(\boldsymbol{\rho}^{\prime \prime}\right)^{-1} \boldsymbol{\rho}^{\prime} & \left(\boldsymbol{\rho}^{\prime \prime}\right)^{-1}
\end{array}\right)
\end{aligned}
$$

are respectively negative definite and positive definite matrices. It follows directly from the variational principles of Gurtin (1964) and Willis (1981), applied to the time harmonic case considered here, that the stationary points of the functionals $Q$ and $R$ correspond to solutions of the elastodynamics equations, in agreement with the results of the previous section. 
When $\boldsymbol{\rho}^{\prime \prime}$ is very small we have

$$
\left(\begin{array}{c}
\underline{\omega}^{\prime} \underline{\mathbf{u}}^{\prime} \\
\left(\mathbf{b}^{\prime}+\nabla \cdot \underline{\boldsymbol{\tau}}^{\prime}\right) / \omega
\end{array}\right) \mathcal{P}\left(\begin{array}{c}
\omega \underline{\mathbf{u}}^{\prime} \\
\left(\mathbf{b}^{\prime}+\nabla \cdot \underline{\boldsymbol{\tau}}^{\prime}\right) / \omega
\end{array}\right) \approx\left[\omega^{2} \boldsymbol{\rho}^{\prime} \underline{\mathbf{u}}^{\prime}+\mathbf{b}^{\prime}+\nabla \cdot \underline{\boldsymbol{\tau}}^{\prime}\right]\left(\omega^{2} \boldsymbol{\rho}^{\prime \prime}\right)^{-1}\left[\omega^{2} \boldsymbol{\rho}^{\prime} \underline{\mathbf{u}}^{\prime}+\mathbf{b}^{\prime}+\nabla \cdot \underline{\boldsymbol{\tau}^{\prime}}\right]
$$

and the variational principles only will be useful if we take trial fields with $\omega^{2} \boldsymbol{\rho}^{\prime} \underline{\mathbf{u}}^{\prime}+\mathbf{b}^{\prime}+$ $\nabla \cdot \boldsymbol{\tau}^{\prime}$ approximately zero. The variational principle involving the functional $Y$ still holds in the limit as $\boldsymbol{\rho}^{\prime \prime} \rightarrow 0$ provided we first choose

$$
\underline{\mathbf{u}}^{\prime}=-\left(\boldsymbol{\rho}^{\prime}\right)^{-1}\left(\mathbf{b}^{\prime}+\nabla \cdot \underline{\boldsymbol{\tau}}^{\prime}\right) / \omega^{2} .
$$

Then, when $\boldsymbol{\rho}$ is real, we have the variational principle

$$
Y\left(\boldsymbol{\tau}^{\prime}\right)=\inf _{\underline{\boldsymbol{\tau}}^{\prime}} Y\left(\underline{\boldsymbol{\tau}^{\prime}}\right)
$$

where

$$
Y\left(\underline{\boldsymbol{\tau}}^{\prime}\right)=\int_{\Gamma}-\left(\begin{array}{c}
\underline{\boldsymbol{\epsilon}}^{\prime} \\
-\underline{\boldsymbol{\tau}}^{\prime}
\end{array}\right) \cdot \mathcal{C}\left(\begin{array}{c}
\underline{\boldsymbol{\epsilon}}^{\prime} \\
-\underline{\boldsymbol{\tau}}^{\prime}
\end{array}\right)+2 \mathbf{b}^{\prime \prime} \cdot \underline{\mathbf{u}}^{\prime},
$$

in which $\underline{\mathbf{u}}^{\prime}$ and $\underline{\boldsymbol{\epsilon}}^{\prime}$ are given by (3.27) and (3.24). In this variational principle the trial field $\underline{\boldsymbol{\tau}}^{\prime}$ can be any real symmetric matrix valued field with prescribed values of $\boldsymbol{\tau}^{\prime} \cdot \mathbf{n}$ and $\boldsymbol{\rho}^{-1} \nabla \cdot \boldsymbol{\tau}^{\prime}$ at the boundary $\partial \Gamma$, where prescribing $\boldsymbol{\rho}^{-1} \nabla \cdot \underline{\boldsymbol{\tau}}^{\prime}$ at $\partial \Gamma$ corresponds to prescribing $\underline{\mathbf{u}}^{\prime}$ according to (3.27). The infimum is attained at a field $\boldsymbol{\tau}^{\prime}$ associated with solutions of the elastodynamic equation satisfying the prescribed boundary conditions.

\section{The saddle point variational principles for electro- magnetism}

A comparison of the continuum elastodynamic equation (3.17) with Maxwell's equations,

$$
\nabla \times \mathbf{E}=i \omega \mathbf{B}, \quad \nabla \times \mathbf{H}=-i \omega \mathbf{D}+\mathbf{j}, \quad \mathbf{D}=\varepsilon \mathbf{E}, \quad \mathbf{B}=\boldsymbol{\mu} \mathbf{H},
$$

which can be rewritten (Milton, Briane, and Willis 2006) in the form

$$
-\nabla \cdot(\mathbf{C} \nabla \mathbf{E})=i \omega \mathbf{j}+\omega^{2} \varepsilon \mathbf{E}
$$

where now

$$
C_{i j k \ell}=e_{i j m} e_{k \ell n}\left\{\boldsymbol{\mu}^{-1}\right\}_{m n},
$$

in which $\mathbf{E}$ is the electric field, $\mathbf{j}$ is the free current $\boldsymbol{\varepsilon}$ the electric permittivity tensor, $\boldsymbol{\mu}$ the magnetic permeability tensor, and $e_{i j m}=1(-1)$ if $(i, j, m)$ is an even (odd) permutation of $(1,2,3)$ and is zero otherwise, suggests that the preceeding analysis should also extend to three-dimensional electromagnetism. Upon multiplying (4.2) by $e^{i \theta}$ and comparing it with (2.12) we can make the identifications

$$
\mathbf{u}=\mathbf{E}, \quad \mathbf{L}=-e^{i \theta} \mathbf{C}, \quad \mathbf{K}=0, \quad \mathbf{M}=e^{i \theta} \omega^{2} \boldsymbol{\varepsilon}, \quad \mathbf{h}=i \omega e^{i \theta} \mathbf{j} .
$$


The problem is now that $\mathbf{C}$ acting upon any symmetric matrix is zero, and so $\mathbf{L}$ and hence $\mathbf{Z}$ are singular, which is a problem for computing the inverses in the equation (2.36) for $\mathcal{L}$. This problem is rectified by replacing $\sqcap$ everywhere with $\widehat{\Pi}$ defined by

$$
\widehat{\Pi} \mathbf{E} \equiv\left(\begin{array}{c}
{\left[\nabla \mathbf{E}-(\nabla \mathbf{E})^{T}\right] / 2} \\
\mathbf{E}
\end{array}\right) .
$$

Then the fields $\mathcal{F}$ and $\mathcal{G}$ have the form (2.1), with $\mathbf{F}$ and $\mathbf{G}$ being antisymmetric $3 \times 3$ matrices: thus $\mathcal{F}$ and $\mathcal{G}$ can be represented by 6 component vectors. We choose $\theta$ such that the imaginary part of

$$
\mathbf{Z}=\left(\begin{array}{cc}
-e^{i \theta} \mathbf{C} & 0 \\
0 & e^{i \theta} \omega^{2} \boldsymbol{\varepsilon}
\end{array}\right)
$$

satisfies (2.15) on the space of these fields, and we compute the inverse of $\mathbf{Z}^{\prime \prime}$ on the space of these fields.

Let us assume, for simplicity, that

$$
\varepsilon^{\prime \prime}(\mathbf{x}) \geq \alpha_{1} \mathbf{I}, \quad \boldsymbol{\mu}^{\prime \prime}(\mathbf{x}) \geq \alpha_{2} \mathbf{I}, \quad \forall \mathbf{x} \in \Gamma,
$$

for some $\alpha_{1}>0$ and $\alpha_{2}>0$. (By multiplying (4.2) by $e^{i \theta}$ and redefining $\boldsymbol{\varepsilon}, \boldsymbol{\mu}$ and $\mathbf{j}$ if necessary.) Then we can take $\theta=0$ and (2.15) holds for some $\alpha>0$. From (4.1) and (4.5) we see that

$$
\mathcal{F}=\widehat{\Pi} \mathbf{E}=\left(\begin{array}{c}
\mathbf{F} \\
\mathbf{E}
\end{array}\right), \quad \text { with } \mathbf{F}=\frac{i \omega}{2}\left(\begin{array}{ccc}
0 & -B_{3} & B_{2} \\
B_{3} & 0 & -B_{1} \\
-B_{2} & B_{1} & 0
\end{array}\right),
$$

and the constitutive law (2.9) implies

$$
\mathcal{G}=\left(\begin{array}{c}
\mathbf{G} \\
\omega^{2} \mathbf{D}
\end{array}\right) \quad \text { with } \mathbf{G}=-i \omega\left(\begin{array}{ccc}
0 & -H_{3} & H_{2} \\
H_{3} & 0 & -H_{1} \\
-H_{2} & H_{1} & 0
\end{array}\right) .
$$

Thus the key property (2.4) reduces to

$$
\int_{\Gamma} \omega^{2}(\mathbf{B} \cdot \mathbf{H}+\mathbf{E} \cdot \mathbf{D})+i \omega \mathbf{j} \cdot \mathbf{E}=\int_{\partial \Gamma}-i \omega(\mathbf{H} \times \mathbf{n}) \cdot \mathbf{E}=\int_{\partial \Gamma}-i \omega(\mathbf{E} \times \mathbf{H}) \cdot \mathbf{n},
$$

and holds for all fields $\mathbf{E}, \mathbf{D}, \mathbf{B}$, and $\mathbf{H}$ satisfying the differential constraints

$$
\nabla \times \mathbf{E}=i \omega \mathbf{B}, \quad \nabla \times \mathbf{H}=-i \omega \mathbf{D}+\mathbf{j} .
$$

Now the first relation (2.20) will hold if

$$
\int_{\partial \Gamma}\left(\mathbf{G}^{\prime \prime} \cdot \mathbf{n}\right) \cdot \mathbf{s}=\int_{\partial \Gamma}-i \omega\left(\mathbf{H}^{\prime \prime} \times \mathbf{n}\right) \cdot \mathbf{s}=0,
$$


and for this it suffices that the tangential component of $\mathbf{s}$ vanishes at the surface $\partial \Gamma$. Similarly the second relation in (2.20) will hold if this condition is satisfied. Therefore the infimum and supremum in (2.17) can be extended to all $\underline{\mathbf{u}}^{\prime}$ and $\underline{\mathbf{u}}^{\prime \prime}$ with the same tangential components as, respectively, $\mathbf{E}^{\prime}$ and $\mathbf{E}^{\prime \prime}$. Similarly in the minimization variational principle (2.41), or (2.51), we only need require that $\underline{\mathbf{u}}^{\prime}$, or $\underline{\mathbf{u}}^{\prime \prime}$, have the same tangential components as $\mathbf{E}^{\prime}$, or $\mathbf{E}^{\prime \prime}$.

With $\sqcap$ replaced by $\widehat{\Pi}$ the analysis of equations $(2.23)-(2.29)$ and $(2.44)-(2.50)$ still holds, and we recover Maxwell's equations from the Euler-Lagrange equations associated with the variational principles. If we introduce the tangential components

$$
\mathbf{E}_{\|}(\mathbf{x})=\mathbf{E}-\mathbf{n}(\mathbf{n} \cdot \mathbf{E}), \quad \mathbf{H}_{\|}(\mathbf{x})=\mathbf{H}-\mathbf{n}(\mathbf{n} \cdot \mathbf{H})
$$

of the fields $\mathbf{E}(\mathbf{x})$ and $\mathbf{H}(\mathbf{x})$ at the boundary $\partial \Gamma$, then

$$
\mathbf{q}_{0}(\mathbf{x})=\mathbf{G} \cdot \mathbf{n}=-i \omega\left(\mathbf{H}_{\|} \times \mathbf{n}\right) .
$$

When $\mathbf{j}=0$ we can write $\mathbf{q}_{0}=\mathbf{N E}_{\|}$, thus defining the map $\mathbf{N}$ which governs the electrodynamic response of the body at the frequency $\omega$. The analysis of (2.63) $-(2.68)$ shows that the map $\mathbf{N}$ is symmetric with positive semidefinite imaginary part, and can be bounded (when $\mathbf{j}=0$ ) using the minimization variational principles.

Equivalently we can find the variational principles directly from Maxwell's equations (4.1). The key property (basically Poynting's theorem) (4.10) is obtained by integrating over $\Gamma$ the identity

$$
\nabla \cdot(\mathbf{E} \times \mathbf{H})=\mathbf{H} \cdot(\nabla \times \mathbf{E})-\mathbf{E} \cdot(\nabla \times \mathbf{H})=i \omega(\mathbf{H} \cdot \mathbf{B}+\mathbf{E} \cdot \mathbf{D})-\mathbf{j} \cdot \mathbf{E}
$$

Motivated by the form of (4.8) and (4.9) let us redefine the fields

$$
\mathcal{F}=\left(\begin{array}{c}
\mathbf{F} \\
\mathbf{f}
\end{array}\right)=\left(\begin{array}{c}
i \omega \mathbf{B} \\
\mathbf{E}
\end{array}\right), \quad \mathcal{G}=\left(\begin{array}{c}
\mathbf{G} \\
\mathbf{g}
\end{array}\right)=\left(\begin{array}{c}
-i \omega \mathbf{H} \\
\omega^{2} \mathbf{D}
\end{array}\right)
$$

which are subject to the differential constraints that

$$
\mathcal{F}=\Omega \mathbf{E}, \quad i \omega \mathbf{j}+\mho \mathcal{G}=0,
$$

where the operators $\Omega$ and $\mho$ are defined by

$$
\Omega \mathbf{E}=\left(\begin{array}{c}
\nabla \times \mathbf{E} \\
\mathbf{E}
\end{array}\right), \quad \mho \mathcal{G}=\nabla \times \mathbf{G}+\mathbf{g}
$$

so that the differential constraints (4.11) and (4.17) are equivalent. The key property (4.10) now takes the form

$$
\int_{\Gamma} \mathcal{G} \cdot \mathcal{F}+i \omega \mathbf{j} \cdot \mathbf{E}=\int_{\partial \Gamma}(\mathbf{E} \times \mathbf{G}) \cdot \mathbf{n}
$$


where $\mathcal{G} \cdot \mathcal{F} \equiv \mathbf{F} \cdot \mathbf{G}+\mathbf{f} \cdot \mathbf{g}$. The constitutive law $\mathcal{G}=\mathbf{Z} \mathcal{F}$ holds with $\mathbf{Z}$ redefined as

$$
\mathbf{Z}=\left(\begin{array}{cc}
-\boldsymbol{\mu}^{-1} & 0 \\
0 & \omega^{2} \boldsymbol{\varepsilon}
\end{array}\right)
$$

and this can be reexpressed in the form (2.14), with

$$
\mathcal{F}^{\prime}=\Omega \mathbf{E}^{\prime}=\left(\begin{array}{c}
-\omega \mathbf{B}^{\prime \prime} \\
\mathbf{E}^{\prime}
\end{array}\right), \quad \mathcal{F}^{\prime \prime}=\Omega \mathbf{E}^{\prime \prime}=\left(\begin{array}{c}
\omega \mathbf{B}^{\prime} \\
\mathbf{E}^{\prime \prime}
\end{array}\right), \quad \mathcal{G}^{\prime}=\left(\begin{array}{c}
\omega \mathbf{H}^{\prime \prime} \\
\omega^{2} \mathbf{D}^{\prime}
\end{array}\right), \quad \mathcal{G}^{\prime \prime}=\left(\begin{array}{c}
-\omega \mathbf{H}^{\prime} \\
\omega^{2} \mathbf{D}^{\prime \prime}
\end{array}\right)
$$

where these fields satisfy the differential constraints

$$
\mathcal{F}^{\prime}=\Omega \mathbf{E}^{\prime}, \quad-\omega \mathbf{j}^{\prime \prime}+\mho \mathcal{S} \mathcal{G}^{\prime}=0, \quad \mathcal{F}^{\prime \prime}=\Omega \mathbf{E}^{\prime \prime}, \quad \omega \mathbf{j}^{\prime}+\mho \mathcal{G} \mathcal{G}^{\prime \prime}=0,
$$

implying, as a corollary of (4.19), that

$$
\int_{\Gamma} \mathcal{G}^{\prime} \cdot \Omega \mathbf{s}-\omega \mathbf{j}^{\prime \prime} \cdot \mathbf{s}=0, \quad \int_{\Gamma} \mathcal{G}^{\prime \prime} \cdot \Omega \mathbf{s}+\omega \mathbf{j}^{\prime} \cdot \mathbf{s}=0
$$

for all real valued vector fields s such that the tangential component of $\mathbf{s}$ vanishes at the surface $\partial \Gamma$. Introducing the functional

$$
\begin{aligned}
Q\left(\underline{\mathbf{E}}^{\prime}, \underline{\mathbf{E}}^{\prime \prime}\right) & =\int_{\Gamma}\left(\begin{array}{c}
\Omega \underline{\mathbf{E}^{\prime}} \\
\Omega \underline{\mathbf{E}^{\prime \prime}}
\end{array}\right) \cdot\left(\begin{array}{cc}
\mathbf{Z}^{\prime \prime} & \mathbf{Z}^{\prime} \\
\mathbf{Z}^{\prime} & -\mathbf{Z}^{\prime \prime}
\end{array}\right)\left(\begin{array}{c}
\Omega \underline{\mathbf{E}}^{\prime} \\
\Omega \underline{\mathbf{E}}^{\prime \prime}
\end{array}\right)+2 \omega\left(\mathbf{j}^{\prime} \cdot \underline{\mathbf{E}^{\prime}}-\mathbf{j}^{\prime \prime} \cdot \underline{\mathbf{E}}^{\prime \prime}\right) \\
& =\int_{\Gamma}[(\Omega \underline{\mathbf{E}}) \cdot \mathbf{Z} \Omega \underline{\mathbf{E}}+2 i \omega \mathbf{j} \cdot \mathbf{E}]^{\prime \prime},
\end{aligned}
$$

we see from (4.23) that

$$
\begin{aligned}
& Q\left(\mathbf{E}^{\prime}+\mathbf{s}, \mathbf{E}^{\prime \prime}\right)=Q\left(\mathbf{E}^{\prime}, \mathbf{E}^{\prime \prime}\right)+\int_{\Gamma}(\Omega \mathbf{s}) \cdot \mathbf{Z}^{\prime \prime} \Omega \mathbf{s}, \\
& Q\left(\mathbf{E}^{\prime}, \mathbf{E}^{\prime \prime}+\mathbf{s}\right)=Q\left(\mathbf{E}^{\prime}, \mathbf{E}^{\prime \prime}\right)-\int_{\Gamma}(\Omega \mathbf{s}) \cdot \mathbf{Z}^{\prime \prime} \Omega \mathbf{s} .
\end{aligned}
$$

Consequently the pair of fields $\left(\underline{\mathbf{E}}^{\prime}, \underline{\mathbf{E}}^{\prime \prime}\right)=\left(\mathbf{E}^{\prime}, \mathbf{E}^{\prime \prime}\right)$ is at the saddle point of (2.17) and of (2.18), where the infimum and supremum are over all vector fields $\underline{\mathbf{E}}^{\prime}$ and $\underline{\mathbf{E}}^{\prime \prime}$ with the same tangential components at the boundary $\partial \Gamma$ as, respectively, $\mathbf{E}^{\prime}$ and $\mathbf{E}^{\prime \prime}$. Due to the diagonal structure (4.20) of the matrix $\mathbf{Z}(\mathbf{x})$ the formula (4.24) for $Q\left(\underline{\mathbf{E}}^{\prime}, \underline{\mathbf{E}}^{\prime \prime}\right)$ reduces to

$$
Q\left(\underline{\mathbf{E}}^{\prime}, \underline{\mathbf{E}}^{\prime \prime}\right)=\int_{\Gamma}\left[(\nabla \times \underline{\mathbf{E}}) \cdot \mathbf{m}(\nabla \times \underline{\mathbf{E}})+\omega^{2} \mathbf{E} \cdot \varepsilon \mathbf{E}+2 i \omega \mathbf{j} \cdot \mathbf{E}\right]^{\prime \prime},
$$

where $\mathbf{m}=-\boldsymbol{\mu}^{-1}$, like $\boldsymbol{\varepsilon}$, has positive definite imaginary part. Hence we have the saddle point variational principles

$$
Q\left(\mathbf{E}^{\prime}, \mathbf{E}^{\prime \prime}\right)=\inf _{\underline{\mathbf{E}}^{\prime}} \sup _{\underline{\mathbf{E}}^{\prime \prime}} Q\left(\underline{\mathbf{E}}^{\prime}, \underline{\mathbf{E}}^{\prime \prime}\right)=\sup _{\underline{\mathbf{E}}^{\prime \prime}} \inf _{\underline{\mathbf{E}}^{\prime}} Q\left(\underline{\mathbf{E}}^{\prime}, \underline{\mathbf{E}}^{\prime \prime}\right),
$$


where

$$
\begin{aligned}
Q\left(\mathbf{E}^{\prime}, \mathbf{E}^{\prime \prime}\right) & =\int_{\Gamma} \mathcal{G}^{\prime \prime} \cdot \mathcal{F}^{\prime}+\mathcal{G}^{\prime} \cdot \mathcal{F}^{\prime \prime}+2 \omega\left(\mathbf{j}^{\prime} \cdot \underline{\mathbf{E}^{\prime}}-\mathbf{j}^{\prime \prime} \cdot \underline{\mathbf{E}^{\prime \prime}}\right) \\
& =\int_{\Gamma} \omega^{2}\left(\mathbf{H}^{\prime} \cdot \mathbf{B}^{\prime \prime}+\mathbf{H}^{\prime \prime} \cdot \mathbf{B}^{\prime}+\mathbf{E}^{\prime} \cdot \mathbf{D}^{\prime \prime}+\mathbf{E}^{\prime \prime} \cdot \mathbf{D}^{\prime}\right)+2 \omega\left(\mathbf{j}^{\prime} \cdot \underline{\mathbf{E}^{\prime}}-\mathbf{j}^{\prime \prime} \cdot \underline{\mathbf{E}^{\prime}}\right) .
\end{aligned}
$$

When $\mathbf{j}=0$ we can use (4.15) to express $Q\left(\mathbf{E}^{\prime}, \mathbf{E}^{\prime \prime}\right)$ just in terms of the tangential components of the fields at the boundary:

$$
Q\left(\mathbf{E}^{\prime}, \mathbf{E}^{\prime \prime}\right)=\omega \int_{\partial \Gamma}\left(\mathbf{E}^{\prime \prime} \times \mathbf{H}^{\prime \prime}\right) \cdot \mathbf{n}-\left(\mathbf{E}^{\prime} \times \mathbf{H}^{\prime}\right) \cdot \mathbf{n} \quad \text { when } \mathbf{j}=0 .
$$

The result that the stationary point of the variational principle (4.27) corresponds to a solution of Maxwell's equations follows from a variational principle derived by Willis (1984). The Willis variational principle is expressed in terms of the electromagnetic potentials $V(\mathbf{x})$ and $\mathbf{A}(\mathbf{x})$, which are associated with $\mathbf{E}$ and $\mathbf{B}$ through the relations

$$
\mathbf{E}=-\nabla V+i \omega \mathbf{A}, \quad \mathbf{B}=\nabla \times \mathbf{A} .
$$

and, in the time harmonic case, involves (to within a proportionality constant) the functional

$$
\widehat{Q}(\underline{V}, \underline{\mathbf{A}})=\omega^{2} \int_{\partial \Gamma}\left\{[-(\nabla \times \underline{\mathbf{A}}) \cdot \mathbf{m}(\nabla \times \underline{\mathbf{A}})+(\nabla \underline{V}-i \omega \underline{\mathbf{A}}) \cdot \varepsilon(\nabla \underline{V}-i \omega \underline{\mathbf{A}})+2 i \underline{V} \nabla \cdot \mathbf{j} / \omega-2 \underline{\mathbf{A}} \cdot \mathbf{j}] e^{-i \omega t}\right\}^{\prime},
$$

where, as usual, the prime denotes the real part. His variational principle states that for any choice of $t$ the functional $\widehat{Q}(\underline{V}, \underline{\mathbf{A}})$ applied to trial fields $\underline{V}$ and $\underline{\mathbf{A}}$ having any given fixed values at the boundary $\partial \Gamma$, has a stationary point at the potentials $V$ and A solving Maxwell's equations, and having the prescribed boundary values. Setting $\underline{\mathbf{E}}=-\nabla \underline{V}+i \omega \underline{\mathbf{A}}$ one sees by integrating by parts that $\widehat{Q}(\underline{V}, \underline{\mathbf{A}})$ is just a functional of $\underline{\mathbf{E}}$ alone, and when $t$ is chosen with $e^{-i \omega t}=-i$ one has the identity

$$
\widehat{Q}(\underline{V}, \underline{\mathbf{A}})=Q\left(\underline{\mathbf{E}}^{\prime}, \underline{\mathbf{E}}^{\prime \prime}\right) \text {. }
$$

Furthermore the values of $\underline{V}$ and $\underline{\mathbf{A}}$ on $\partial \Gamma$ determine the tangential (but not normal) components of $\underline{\mathbf{E}}^{\prime}$ and $\underline{\mathbf{E}}^{\prime \prime}$ on $\partial \Gamma$.

There is also a dual variational principle. By taking real and imaginary parts of the constitutive relation $\mathcal{F}=\mathbf{K} \mathcal{G}$ where $\mathbf{K}=\mathbf{Z}^{-1}$ has negative definite imaginary part we obtain (2.23). Let us introduce the functional

$$
R\left(\underline{\mathbf{H}}^{\prime}, \underline{\mathbf{H}}^{\prime \prime}\right)=\int_{\Gamma}\left(\begin{array}{c}
\underline{\mathcal{G}}^{\prime} \\
\underline{\mathcal{G}}^{\prime \prime}
\end{array}\right)\left(\begin{array}{cc}
\mathbf{K}^{\prime \prime} & \mathbf{K}^{\prime} \\
\mathbf{K}^{\prime} & -\mathbf{K}^{\prime \prime}
\end{array}\right)\left(\begin{array}{l}
\underline{\mathcal{G}}^{\prime} \\
\underline{\mathcal{G}}^{\prime \prime}
\end{array}\right)=\int_{\Gamma}(\underline{\mathcal{G}} \cdot \mathbf{K} \underline{\mathcal{G}})^{\prime \prime},
$$


where $\underline{\mathcal{G}}^{\prime}$ and $\underline{\mathcal{G}}^{\prime \prime}$ are given by

$$
\underline{\mathcal{G}}^{\prime}=\omega\left(\begin{array}{c}
\mathbf{j}^{\prime \prime}-\underline{\mathbf{H}}^{\prime \prime} \\
\nabla \\
\underline{\mathbf{H}}^{\prime \prime}
\end{array}\right), \quad \underline{\mathcal{G}}^{\prime \prime}=\omega\left(\begin{array}{c}
-\underline{\mathbf{H}}^{\prime} \\
-\mathbf{j}^{\prime}+\bar{\nabla} \times \underline{\mathbf{H}}^{\prime}
\end{array}\right),
$$

(to ensure that $-\omega \mathbf{j}^{\prime \prime}+\mho \widetilde{\mathcal{G}^{\prime}}=0$ and $\omega \mathbf{j}^{\prime}+\mho \delta \underline{\mathcal{G}}^{\prime \prime}=0$ inside $\Gamma$ ) and $\underline{\mathbf{H}}^{\prime \prime}$ and $\underline{\mathbf{H}}^{\prime}$ have the same tangential components as, respectively, $\overline{\mathbf{H}}^{\prime \prime}$ and $\mathbf{H}^{\prime}$. We then have the saddle point variational principles

$$
R\left(\mathbf{H}^{\prime}, \mathbf{H}^{\prime \prime}\right)=\sup _{\underline{\mathbf{H}}^{\prime \prime} \underline{\mathbf{H}}^{\prime}} R\left(\underline{\mathbf{H}}^{\prime}, \underline{\mathbf{H}}^{\prime \prime}\right)=\inf _{\underline{\mathbf{H}}^{\prime}} \sup _{\underline{\mathbf{H}}^{\prime \prime}} R\left(\underline{\mathbf{H}}^{\prime}, \underline{\mathbf{H}}^{\prime \prime}\right),
$$

where the supremum and infimum are over fields with the required tangential components at the boundary. Due to the diagonal structure (4.20) of $\mathbf{Z}(\mathbf{x})$ the formula for $R\left(\underline{\mathbf{H}}^{\prime}, \underline{\mathbf{H}}^{\prime \prime}\right)$ reduces to

$$
R\left(\underline{\mathbf{H}}^{\prime}, \underline{\mathbf{H}}^{\prime \prime}\right)=\int_{\Gamma}\left[(\mathbf{j}-\nabla \times \underline{\mathbf{H}}) \cdot \mathbf{e}(\mathbf{j}-\nabla \times \underline{\mathbf{H}})+\omega^{2} \underline{\mathbf{H}} \boldsymbol{\mu} \underline{\mathbf{H}}\right]^{\prime \prime},
$$

where $\mathbf{e}=-\boldsymbol{\varepsilon}^{-1}$, like $\boldsymbol{\mu}$, has positive definite imaginary part. From this expression one can see that $R\left(\underline{\mathbf{H}}^{\prime}, \underline{\mathbf{H}}^{\prime \prime}\right)$ is a special case of the functional associated with the Willis (1984) dual variational principle. We also have that

$$
R\left(\mathbf{H}^{\prime}, \mathbf{H}^{\prime \prime}\right)=\int_{\Gamma} \mathcal{G}^{\prime \prime} \cdot \mathcal{F}^{\prime}+\mathcal{G}^{\prime} \cdot \mathcal{F}^{\prime \prime}=\int_{\Gamma} \omega^{2}\left(\mathbf{H}^{\prime} \cdot \mathbf{B}^{\prime \prime}+\mathbf{H}^{\prime \prime} \cdot \mathbf{B}^{\prime}+\mathbf{E}^{\prime} \cdot \mathbf{D}^{\prime \prime}+\mathbf{E}^{\prime \prime} \cdot \mathbf{D}^{\prime}\right),
$$

which when $\mathbf{j}=0$ can be expressed in terms of the tangential components of the fields at the boundary:

$$
R\left(\mathbf{H}^{\prime}, \mathbf{H}^{\prime \prime}\right)=\omega \int_{\partial \Gamma}\left(\mathbf{E}^{\prime \prime} \times \mathbf{H}^{\prime \prime}\right) \cdot \mathbf{n}-\left(\mathbf{E}^{\prime} \times \mathbf{H}^{\prime}\right) \cdot \mathbf{n} \quad \text { when } \mathbf{j}=0 .
$$

Consider a medium which is locally isotropic and such that $\varepsilon(\mathbf{x})$ is purely imaginary (which may be a good approximation at low frequencies in conductive media) while $\boldsymbol{\mu}(\mathbf{x})$ is purely real (and so (4.7) only holds in the limiting sense with $\alpha_{2}=0$ ). We can write

$$
\boldsymbol{\varepsilon}=i \mathbf{I} /(\omega \rho), \quad \mathbf{e}=-i \omega \rho \mathbf{I}, \quad \boldsymbol{\mu}=\mu \mathbf{I},
$$

where the resistivity $\rho(\mathbf{x})$ and the permeability $\mu(\mathbf{x})$ are purely real and positive. Then the expression (4.36) reduces to

$$
R\left(\underline{\mathbf{H}}^{\prime}, \underline{\mathbf{H}}^{\prime \prime}\right)=\int_{\Gamma} \omega \rho\left(\left|\mathbf{j}^{\prime}-\nabla \times \underline{\mathbf{H}}^{\prime}\right|^{2}-\left|\mathbf{j}^{\prime \prime}-\nabla \times \underline{\mathbf{H}}^{\prime \prime}\right|^{2}\right)+2 \omega^{2} \mu \underline{\mathbf{H}}^{\prime} \cdot \underline{\mathbf{H}}^{\prime \prime}
$$

and then when $\mathbf{j}=0$ the saddle point variational principle (4.35) corresponds to the Borcea (1999) variational principle. (Note that $\mathbf{j}$ has a different meaning in that paper.) Now Maxwell's equations still hold if we make the replacements

$$
\varepsilon \rightarrow-i \varepsilon, \quad \boldsymbol{\mu} \rightarrow i \boldsymbol{\mu}, \quad \mathbf{E} \rightarrow i \mathbf{E}, \quad \mathbf{B} \rightarrow i \mathbf{B}, \quad \mathbf{D} \rightarrow \mathbf{D}, \quad \mathbf{H} \rightarrow \mathbf{H}, \quad \mathbf{j} \rightarrow \mathbf{j}
$$


(which is a special case of the transformation (2.57)) and with these replacements applied to (4.39) the expression (4.36) reduces to

$$
R\left(\underline{\mathbf{H}}^{\prime}, \underline{\mathbf{H}}^{\prime \prime}\right)=\int_{\Gamma}-2 \omega \rho\left(\mathbf{j}^{\prime}-\nabla \times \underline{\mathbf{H}}^{\prime}\right) \cdot\left(\mathbf{j}^{\prime \prime}-\nabla \times \underline{\mathbf{H}}^{\prime \prime}\right)+\omega^{2} \mu\left(\left|\underline{\mathbf{H}}^{\prime}\right|^{2}-\left|\underline{\mathbf{H}}^{\prime \prime}\right|^{2}\right),
$$

and when $\mathbf{j}=0$ the saddle point variational principle (4.35) then corresponds to the other Borcea variational principle. Multiplying $\varepsilon$ and dividing $\boldsymbol{\mu}$ by $e^{i \theta}$, where $0>\theta>-\pi / 2$ leads to a continuous family of saddle point variational principles which interpolate the two Borcea variational principles.

\section{Minimization variational principles for electromag- netism}

To obtain a minimization principle we rewrite the constitutive law in the form (2.35) where $\mathcal{L}$ is given by (2.36). Then we introduce the quadratic functional

$$
Y\left(\underline{\mathbf{E}}^{\prime}, \underline{\mathbf{H}}^{\prime \prime}\right)=\int_{\Gamma}\left(\begin{array}{l}
\Omega \underline{\mathbf{E}}^{\prime} \\
-\underline{\mathcal{G}}^{\prime}
\end{array}\right) \cdot \mathcal{L}\left(\begin{array}{l}
\Omega \underline{\mathbf{E}}^{\prime} \\
-\underline{\mathcal{G}}^{\prime}
\end{array}\right)+2 \omega \mathbf{j}^{\prime} \cdot \underline{\mathbf{E}}^{\prime},
$$

where $\underline{\mathcal{G}}^{\prime}$ is given by

$$
\underline{\mathcal{G}}^{\prime}=\omega\left(\mathbf{j}^{\prime \prime}-\underline{\mathbf{H}}^{\prime \prime} \times \underline{\mathbf{H}}^{\prime \prime}\right)
$$

(to ensure that $-\omega \mathbf{j}^{\prime \prime}+\mho \underline{\mathcal{G}}^{\prime}=0$ inside $\Gamma$ ) and $\underline{\mathbf{E}}^{\prime}$ and $\underline{\mathbf{H}}^{\prime \prime}$ have the same tangential components at the boundary $\partial \Gamma$ as, respectively, $\mathbf{E}^{\prime}$ and $\omega \mathbf{H}^{\prime \prime}$. Given two vector fields $\mathbf{s}$ and $\mathbf{t}$ with zero tangential components at the boundary $\partial \Gamma$ then (4.23) and the key property (4.19) imply

$$
\int_{\Gamma} \mathcal{G}^{\prime \prime} \cdot(\Omega \mathbf{s})+\omega \mathbf{j}^{\prime} \cdot \mathbf{s}-\mathcal{T} \cdot\left(\Omega \mathbf{u}^{\prime \prime}\right)=0, \quad \text { where } \mathcal{T}=\omega\left(\begin{array}{c}
\mathbf{t} \\
-\nabla \times \mathbf{t}
\end{array}\right) .
$$

As a result we deduce that

$$
Y\left(\mathbf{E}^{\prime}+\mathbf{s}, \mathbf{H}^{\prime \prime}+\mathbf{t}\right)=Y\left(\mathbf{E}^{\prime}, \mathbf{H}^{\prime \prime}\right)+\int_{\Gamma}\left(\begin{array}{c}
\Omega \mathbf{s} \\
-\mathcal{T}
\end{array}\right) \cdot \mathcal{L}\left(\begin{array}{c}
\Omega \mathbf{s} \\
-\mathcal{T}
\end{array}\right)
$$

Consequently, because $\mathcal{L}$ is positive definite under the assumption (4.7), we the minimization variational principle

$$
Y\left(\mathbf{E}^{\prime}, \mathbf{H}^{\prime \prime}\right)=\inf _{\underline{\mathbf{E}}^{\prime}} \inf _{\underline{\mathbf{H}}^{\prime \prime}} Y\left(\underline{\mathbf{E}}^{\prime}, \underline{\mathbf{H}}^{\prime \prime}\right),
$$

where the infimums are over vector fields $\underline{\mathbf{E}}^{\prime}$ and $\underline{\mathbf{H}}^{\prime \prime}$ with the same tangential components at the boundary $\partial \Gamma$ as, respectively, $\mathbf{E}^{\prime}$ and $\mathbf{H}^{\prime \prime}$. Using (2.37) the minimum value of $Y\left(\underline{\mathbf{E}}^{\prime}, \underline{\mathbf{H}}^{\prime \prime}\right)$ is

$$
Y\left(\mathbf{E}^{\prime}, \mathbf{H}^{\prime \prime}\right)=\int_{\Gamma} \omega^{2}\left(\mathbf{H}^{\prime} \cdot \mathbf{B}^{\prime \prime}-\mathbf{H}^{\prime \prime} \cdot \mathbf{B}^{\prime}+\mathbf{E}^{\prime} \cdot \mathbf{D}^{\prime \prime}-\mathbf{E}^{\prime \prime} \cdot \mathbf{D}^{\prime}\right)+2 \omega \mathbf{j}^{\prime} \cdot \underline{\mathbf{E}^{\prime}} .
$$


Now the power dissipated into heat in the body $\Gamma$, averaged over a cycle of oscillation is $W(\mathbf{E}, \mathbf{H})$

$$
\begin{aligned}
& =\int_{\Gamma}\left[\frac{\omega}{2 \pi} \int_{0}^{2 \pi}\left(\mathbf{E} e^{-i \omega t}\right)^{\prime} \cdot \frac{\partial\left(\mathbf{D} e^{-i \omega t}\right)^{\prime}}{\partial t}+\left(\mathbf{H} e^{-i \omega t}\right)^{\prime} \cdot \frac{\partial\left(\mathbf{B} e^{-i \omega t}\right)^{\prime}}{\partial t}+\left(\mathbf{E} e^{-i \omega t}\right)^{\prime} \cdot\left(\mathbf{j} e^{-i \omega t}\right)^{\prime} d t\right] \\
& =\frac{1}{2} \int_{\Gamma} \omega\left(\mathbf{H}^{\prime} \cdot \mathbf{B}^{\prime \prime}-\mathbf{H}^{\prime \prime} \cdot \mathbf{B}^{\prime}+\mathbf{E}^{\prime} \cdot \mathbf{D}^{\prime \prime}-\mathbf{E}^{\prime \prime} \cdot \mathbf{D}^{\prime}\right)+\mathbf{E}^{\prime} \cdot \mathbf{j}^{\prime}+\mathbf{E}^{\prime \prime} \cdot \mathbf{j}^{\prime \prime}
\end{aligned}
$$

which is proportional to (5.6) when $\mathbf{j}=0$. So the minimum value of $Y$ has a physical interpretation when $\mathbf{j}=0$.

Due to the diagonal structure (4.20) of $\mathbf{Z}$ the formula (5.1) reduces to

$$
\begin{aligned}
& \left.Y\left(\underline{\mathbf{E}}^{\prime}, \underline{\mathbf{H}}^{\prime \prime}\right)=\int_{\Gamma}\left(\begin{array}{c}
\omega \underline{\mathbf{E}}^{\prime} \\
\nabla \times \underline{\mathbf{H}}^{\prime \prime}
\end{array}\right) \cdot \mathcal{\mathbf { j } ^ { \prime \prime }}\right) \cdot \mathcal{E}\left(\begin{array}{c}
\omega \underline{\mathbf{E}}^{\prime} \\
\nabla \times \underline{\mathbf{H}}^{\prime \prime}-\mathbf{j}^{\prime \prime}
\end{array}\right) \\
& +\left(\begin{array}{c}
\nabla \times \underline{\mathbf{E}^{\prime}} \\
-\omega \underline{\mathbf{H}}^{\prime \prime}
\end{array}\right) \cdot \mathcal{M}\left(\begin{array}{l}
\nabla \times \underline{\mathbf{E}}^{\prime} \\
-\omega \underline{\mathbf{H}}^{\prime \prime}
\end{array}\right)+2 \omega \mathbf{j}^{\prime} \cdot \underline{\mathbf{E}}^{\prime},
\end{aligned}
$$

where $\mathcal{E}(\mathbf{x})$ and $\mathcal{M}(\mathbf{x})$ are the positive definite matrices

$$
\begin{aligned}
\mathcal{E} & =\left(\begin{array}{cc}
\varepsilon^{\prime \prime}+\varepsilon^{\prime}\left(\varepsilon^{\prime \prime}\right)^{-1} \varepsilon^{\prime} & \varepsilon^{\prime}\left(\varepsilon^{\prime \prime}\right)^{-1} \\
\left(\varepsilon^{\prime \prime}\right)^{-1} \varepsilon^{\prime} & \left(\varepsilon^{\prime \prime}\right)^{-1}
\end{array}\right) \\
\mathcal{M} & =\left(\begin{array}{cc}
\mathbf{m}^{\prime \prime}+\mathbf{m}^{\prime}\left(\mathbf{m}^{\prime \prime}\right)^{-1} \mathbf{m}^{\prime} & \mathbf{m}^{\prime}\left(\mathbf{m}^{\prime \prime}\right)^{-1} \\
\left(\mathbf{m}^{\prime \prime}\right)^{-1} \mathbf{m}^{\prime} & \left(\mathbf{m}^{\prime \prime}\right)^{-1}
\end{array}\right) .
\end{aligned}
$$

in which $\mathbf{m}=-\boldsymbol{\mu}^{-1}$. Also the constitutive law (2.35) reduces to

$$
\left(\begin{array}{c}
\mathbf{D}^{\prime \prime} \\
\mathbf{E}^{\prime \prime}
\end{array}\right)=\mathcal{E}\left(\begin{array}{c}
\mathbf{E}^{\prime} \\
-\mathbf{D}^{\prime}
\end{array}\right), \quad\left(\begin{array}{c}
\mathbf{H}^{\prime} \\
-\mathbf{B}^{\prime}
\end{array}\right)=\mathcal{M}\left(\begin{array}{c}
\mathbf{B}^{\prime \prime} \\
\mathbf{H}^{\prime \prime}
\end{array}\right) .
$$

So given any arbitrary prescribed values for the tangential components $\underline{\mathbf{E}}^{\prime}$ and $\underline{\mathbf{H}}^{\prime \prime}$ at the boundary $\partial \Gamma$ the minimum in the variational principle (5.5) will satisfy the EulerLagrange equation and therefore will correspond to a solution of Maxwell equations. From the minimizing fields $\mathbf{E}^{\prime}$ and $\mathbf{H}^{\prime \prime}$ one can, according to (5.10), determine

$$
\begin{aligned}
& \mathbf{E}^{\prime \prime}=\left(\varepsilon^{\prime \prime}\right)^{-1} \varepsilon^{\prime} \mathbf{E}^{\prime}+\left(\varepsilon^{\prime \prime}\right)^{-1}\left(\nabla \times \underline{\mathbf{H}}^{\prime \prime}-\mathbf{j}^{\prime \prime}\right) / \omega, \\
& \mathbf{H}^{\prime}=\mathbf{m}^{\prime}\left(\mathbf{m}^{\prime \prime}\right)^{-1} \mathbf{H}^{\prime \prime}-\left[\mathbf{m}^{\prime \prime}+\mathbf{m}^{\prime}\left(\mathbf{m}^{\prime \prime}\right)^{-1} \mathbf{m}^{\prime}\right]\left(\nabla \times \underline{\mathbf{E}}^{\prime}\right) / \omega,
\end{aligned}
$$

and in particular one can determine the tangential components of these fields at the boundary.

It is often the case that $\boldsymbol{\mu}$ is real, while $\boldsymbol{\varepsilon}$ has a positive definite imaginary part. In this event we redefine $\mathbf{Z}, \mathcal{G}$ and $\mathbf{j}$ by multiplying them by $e^{i \theta}$. Again there is a continuous two-parameter family of related variational principles. We rewrite Maxwell's equations (4.1) in the form

$$
\nabla \times \widetilde{\mathbf{E}}=i \omega \widetilde{\mathbf{B}}, \quad \nabla \times \widetilde{\mathbf{H}}=-i \omega \widetilde{\mathbf{D}}+\widetilde{\mathbf{j}}, \quad \widetilde{\mathbf{D}}=\widetilde{\varepsilon} \widetilde{\mathbf{E}}, \quad \widetilde{\mathbf{B}}=\widetilde{\boldsymbol{\mu}} \widetilde{\mathbf{H}}
$$


where

$$
\begin{aligned}
\widetilde{\mathbf{E}} & =e^{-i \omega t_{0}} \mathbf{E}, \quad \widetilde{\mathbf{D}}=e^{i\left(\theta-\omega t_{0}\right)} \mathbf{D}, \quad \widetilde{\mathbf{H}}=e^{i\left(\theta-\omega t_{0}\right)} \mathbf{H}, \quad \widetilde{\mathbf{B}}=e^{-i \omega t_{0}} \mathbf{B}, \\
\widetilde{\mathbf{j}} & =e^{i\left(\theta-\omega t_{0}\right)} \mathbf{j}, \quad \widetilde{\boldsymbol{\varepsilon}}=e^{i \theta} \boldsymbol{\varepsilon}, \quad \widetilde{\boldsymbol{\mu}}=e^{-i \theta} \boldsymbol{\mu},
\end{aligned}
$$

in which the real constant $t_{0}$ (which may be viewed as a time interval) can be chosen freely, while $\theta$ must be chosen so that

$$
\widetilde{\boldsymbol{\varepsilon}}^{\prime \prime}(\mathbf{x}) \geq \alpha_{1} \mathbf{I}, \quad \widetilde{\boldsymbol{\mu}}^{\prime \prime}(\mathbf{x}) \geq \alpha_{2} \mathbf{I}, \quad \forall \mathbf{x} \in \Gamma,
$$

for some $\alpha_{1}>0$ and $\alpha_{2}>0$. Then the fields with the tildes on them also satisfy Maxwell's equations and the variational principles directly apply to them. With the subsequent replacement (5.13) we obtain a new set of variational principles parameterized by $\theta$ and $t_{0}$. When $\theta$ is non-zero the minimum value of the minimization principles no longer has a physical interpretation as the time averaged power dissipation in the body.

In particular by choosing $\theta=0$ and $t_{0}$ with $e^{-i \omega t_{0}}=-i$ we recover the dual variational principle that

$$
\begin{aligned}
\widetilde{Y}\left(\underline{\mathbf{E}}^{\prime \prime}, \underline{\mathbf{H}}^{\prime}\right)= & \int_{\Gamma}\left(\begin{array}{c}
\omega \underline{\mathbf{E}}^{\prime \prime} \\
\mathbf{j}^{\prime}-\nabla \times \underline{\mathbf{H}}^{\prime}
\end{array}\right) \cdot \mathcal{E}\left(\begin{array}{c}
\omega \underline{\mathbf{E}}^{\prime \prime} \\
\mathbf{j}^{\prime}-\nabla \times \underline{\mathbf{H}}^{\prime}
\end{array}\right) \\
& +\left(\begin{array}{c}
\nabla \times \underline{\mathbf{E}}^{\prime \prime} \\
\omega \underline{\mathbf{H}}^{\prime}
\end{array}\right) \cdot \mathcal{M}\left(\begin{array}{c}
\nabla \times \underline{\mathbf{E}}^{\prime \prime} \\
\omega \underline{\mathbf{H}}^{\prime}
\end{array}\right)+2 \omega \mathbf{j}^{\prime \prime} \cdot \underline{\mathbf{E}}^{\prime \prime},
\end{aligned}
$$

is minimized at $\underline{\mathbf{E}}^{\prime \prime}=\mathbf{E}^{\prime \prime}$ and $\underline{\mathbf{H}}^{\prime}=\mathbf{H}^{\prime}$, under the constraint that $\underline{\mathbf{E}}^{\prime \prime}$ and $\underline{\mathbf{H}}^{\prime}$ have the same tangential values as, respectively, $\mathbf{E}^{\prime \prime}$ and $\mathbf{H}^{\prime}$. A corollary is that

$$
W(\underline{\mathbf{E}}, \underline{\mathbf{H}}) \equiv\left[Y\left(\underline{\mathbf{E}}^{\prime}, \underline{\mathbf{H}}^{\prime \prime}\right)+\widetilde{Y}\left(\underline{\mathbf{E}}^{\prime \prime}, \underline{\mathbf{H}}^{\prime}\right)\right] /(4 \omega)
$$

is minimized at $\underline{\mathbf{E}}=\underline{\mathbf{E}}^{\prime}+i \underline{\mathbf{E}}^{\prime \prime}=\mathbf{E}$ and $\underline{\mathbf{H}}=\underline{\mathbf{H}}^{\prime}+i \underline{\mathbf{H}}^{\prime \prime}=\mathbf{H}$, under the constraint that $\underline{\mathbf{E}}$ and $\underline{\mathbf{H}}$ have the same tangential values as, respectively $\mathbf{E}$ and $\mathbf{H}$. By making the substitutions (5.13) in (5.6) it is easy to check that the minimum value of $W(\underline{\mathbf{E}}, \underline{\mathbf{H}})$ is the average power dissipation $W(\mathbf{E}, \mathbf{H})$ given by (5.7), even when $\mathbf{j}$ is non-zero. An appealing feature of the expression (4.39) is that it remains invariant under the replacement $\underline{\mathbf{E}} \rightarrow e^{-i \omega t_{0}} \underline{\mathbf{E}}, \underline{\mathbf{H}} \rightarrow e^{-i \omega t_{0}} \underline{\mathbf{H}}$ for any real value of $t_{0}$. However a disadvantage is that the boundary conditions are overprescribed: if one imposes boundary conditions on the tangential values of $\underline{\mathbf{E}}$ and $\underline{\mathbf{H}}$ that are not compatible with the moduli $\boldsymbol{\varepsilon}(\mathbf{x})$ and $\boldsymbol{\mu}(\mathbf{x})$ then the fields which minimize $W(\underline{\mathbf{E}}, \underline{\mathbf{H}})$ will not satisfy Maxwell's equations.

When $\boldsymbol{\mu}^{\prime \prime}$, or equivalently $\mathbf{m}^{\prime \prime}$, is very small we have

$$
\left(\begin{array}{c}
\nabla \times \underline{\mathbf{E}^{\prime}} \\
-\omega \underline{\mathbf{H}}^{\prime \prime}
\end{array}\right) \cdot \mathcal{M}\left(\begin{array}{c}
\nabla \times \underline{\mathbf{E}}^{\prime} \\
-\omega \underline{\mathbf{H}}^{\prime \prime}
\end{array}\right) \approx\left[\mathbf{m}^{\prime} \nabla \times \underline{\mathbf{E}}^{\prime}-\omega \underline{\mathbf{H}}^{\prime \prime}\right] \cdot\left(\mathbf{m}^{\prime \prime}\right)^{-1}\left[\mathbf{m}^{\prime} \nabla \times \underline{\mathbf{E}}^{\prime}-\omega \underline{\mathbf{H}}^{\prime \prime}\right]
$$

and the variational principle is only useful if we take trial fields with $\underline{\mathbf{H}}^{\prime \prime} \approx \mathbf{m}^{\prime} \nabla \times \underline{\mathbf{E}}^{\prime} / \omega$. If we take $\underline{\mathbf{H}}^{\prime \prime}=\mathbf{m}^{\prime} \nabla \times \underline{\mathbf{E}}^{\prime} / \omega$ we still obtain a useful variational principle in the limit when $\boldsymbol{\mu}$ is real. From (5.5) and (5.8) we have

$$
Y\left(\mathbf{E}^{\prime}\right)=\inf _{\underline{\mathbf{E}}^{\prime}} Y\left(\underline{\mathbf{E}}^{\prime}\right),
$$


where

$Y\left(\underline{\mathbf{E}}^{\prime}\right)=\int_{\Gamma}\left(\begin{array}{c}\omega \underline{\mathbf{E}}^{\prime} \\ -\nabla \times \boldsymbol{\mu}^{-1}\left(\nabla \times \underline{\mathbf{E}}^{\prime}\right) / \omega-\mathbf{j}^{\prime \prime}\end{array}\right) \cdot \mathcal{E}\left(\begin{array}{c}\omega \underline{\mathbf{E}}^{\prime} \\ -\nabla \times \boldsymbol{\mu}^{-1}\left(\nabla \times \underline{\mathbf{E}}^{\prime}\right) / \omega-\mathbf{j}^{\prime \prime}\end{array}\right)+2 \omega \mathbf{j}^{\prime} \cdot \underline{\mathbf{E}}^{\prime}$.

In this variational principle the infimum is over vector fields $\underline{\mathbf{E}}^{\prime}$ with prescribed tangential components of $\underline{\mathbf{E}}^{\prime}$ and $\boldsymbol{\mu}^{-1} \nabla \times \underline{\mathbf{E}}^{\prime}$ at the boundary $\partial \Gamma$. The infimum is attained at a field $\mathbf{E}^{\prime}$ associated with the solution of Maxwell's equations satisfying the prescribed boundary conditions.

\section{Application to Electromagnetic Tomography}

Here we consider the tomography problem: how can one recover information about the functions $\varepsilon(\mathbf{x})$ and $\boldsymbol{\mu}(\mathbf{x})$ given measurements of the tangential components $\mathbf{E}_{\|}(\mathbf{x})$ and $\mathbf{H}_{\|}(\mathbf{x})$ of the fields $\mathbf{E}(\mathbf{x})$ and $\mathbf{H}(\mathbf{x})$ at the boundary $\partial \Gamma$ ? When it is known that the body $\Gamma$ consists of small inclusions in a matrix this question has been answered by Ammari, Vogelius, and Volkov (2001): see also the review by Ammari and Kang (2004). For other geometries a different approach is needed and the variational principles provide this. First observe from (4.15) and (5.7) that the power dissipation $W(\mathbf{E}, \mathbf{H})$, only depends on the tangential values of these fields at the boundary

$$
W(\mathbf{E}, \mathbf{H})=-\frac{1}{2} \int_{\partial \Gamma}\left(\mathbf{E}_{\|}^{\prime} \times \mathbf{H}_{\|}^{\prime}\right) \cdot \mathbf{n}+\left(\mathbf{E}_{\|}^{\prime \prime} \times \mathbf{H}_{\|}^{\prime \prime}\right) \cdot \mathbf{n},
$$

and, as could be expected physically, is the time-averaged value of the flux of the Poynting vector into the body $\Gamma$. Thus when $\mathbf{j}=0$, and trial fields $\underline{\mathbf{E}}^{\prime}$ and $\underline{\mathbf{H}}^{\prime \prime}$ are chosen having the same tangential values at the boundary as, respectively $\mathbf{E}^{\prime}$ and $\mathbf{H}^{\prime \prime}$, the inequality

$$
W(\mathbf{E}, \mathbf{H}) \leq Y\left(\underline{\mathbf{E}}^{\prime}, \underline{\mathbf{H}}^{\prime \prime}\right) /(2 \omega)
$$

provides for a given $\boldsymbol{\varepsilon}(\mathbf{x})$ and $\boldsymbol{\mu}(\mathbf{x})$ a bound on the possible tangential components of the fields $\underline{\mathbf{E}}^{\prime \prime}$ and $\underline{\mathbf{H}}^{\prime}$. Conversely if these tangential components have been measured the inequality provides one constraint on the possible values of $\boldsymbol{\varepsilon}(\mathbf{x})$ and $\boldsymbol{\mu}(\mathbf{x})$. Additional inequalities on $\boldsymbol{\varepsilon}(\mathbf{x})$ and $\boldsymbol{\mu}(\mathbf{x})$ may be obtained by chosing different trial fields, by working with other minimization variational principles in the family parameterized by $t_{0}$ and $\theta$, and by conducting a series of physical experiments with various different sets of values of the tangential components of the fields $\mathbf{E}(\mathbf{x})$ and $\mathbf{H}(\mathbf{x})$ at the boundary $\partial \Gamma$. It is not necessary that the moduli $\boldsymbol{\varepsilon}(\mathbf{x})$ and $\boldsymbol{\mu}(\mathbf{x})$ remain the same for each physical experiment provided we have some model for how they vary from experiment to experiment. For example, the frequency $\omega$ could be different for the different experiments if we know that the permittivity has the form $\varepsilon(\mathbf{x})=\varepsilon_{0}+i \boldsymbol{\sigma}_{o} / \omega$ (or some other known parametric dependence on $\omega)$. The objective is then to constrain the possible values of the real functions $\varepsilon_{0}(\mathbf{x})$ and $\boldsymbol{\sigma}_{o}(\mathbf{x})$. 
One criticism of this approach is that it only utilizes information (in the case $\theta=0$ ) about the time-averaged power dissipation in the body $\Gamma$. When $t=\theta=0$ the measured values of the functions $\mathbf{E}^{\prime \prime}$ and $\mathbf{H}^{\prime}$ around the boundary $\partial \Gamma$ are not used except to calculate the single scalar quantity $W(\mathbf{E}, \mathbf{H})$. This seems like a tremendous waste of information. However some extra information from the measured fields can be incorporated if one has measurements of the boundary value fields $\mathbf{E}_{\|}^{(j)}(\mathbf{x})$ and $\mathbf{H}_{\|}^{(j)}(\mathbf{x})$ for $n$ experiments indexed by the integer $j=1,2, \ldots, n$, with the moduli $\boldsymbol{\varepsilon}(\mathbf{x})$ and $\boldsymbol{\mu}(\mathbf{x})$ remaining the same for each experiment. Then one can infer the boundary values $\mathbf{E}_{\|}$and $\mathbf{H}_{\|}$associated with a linear combination of these fields:

$$
\mathbf{E}_{\|}=\sum_{j=1}^{n} \lambda_{j} \mathbf{E}_{\|}^{(j)}, \quad \mathbf{H}_{\|}=\sum_{j=1}^{n} \lambda_{j} \mathbf{H}_{\|}^{(j)},
$$

where $\lambda_{1}, \lambda_{2}, \ldots \lambda_{n}$ are a set of complex constants. Letting $\mathbf{E}$ and $\mathbf{H}$ denote the corresponding electric and magnetic fields inside $\Gamma$, it follows that $W(\mathbf{E}, \mathbf{H})$ can be expressed as the sum

$$
\begin{aligned}
W(\mathbf{E}, \mathbf{H}) & =\sum_{j=1}^{n} \sum_{k=1}^{n}\left(\lambda_{j}^{\prime} \lambda_{k}^{\prime}+\lambda_{j}^{\prime \prime} \lambda_{k}^{\prime \prime}\right) W_{j k}+\left(\lambda_{j}^{\prime \prime} \lambda_{k}^{\prime}-\lambda_{k}^{\prime \prime} \lambda_{j}^{\prime}\right) S_{j k} \\
& =\frac{1}{2} \sum_{j=1}^{n} \sum_{k=1}^{n}\left(\lambda_{j}^{\prime} \lambda_{k}^{\prime}+\lambda_{j}^{\prime \prime} \lambda_{k}^{\prime \prime}\right)\left(W_{j k}+W_{k j}\right)+\left(\lambda_{j}^{\prime \prime} \lambda_{k}^{\prime}-\lambda_{k}^{\prime \prime} \lambda_{j}^{\prime}\right)\left(S_{j k}-S_{k j}\right)
\end{aligned}
$$

involving the real quantities

$$
\begin{aligned}
W_{j k} & =-\frac{1}{2} \int_{\partial \Gamma}\left(\mathbf{E}_{\|}^{\prime(j)} \times \mathbf{H}_{\|}^{\prime(k)}\right) \cdot \mathbf{n}+\left(\mathbf{E}_{\|}^{\prime \prime(j)} \times \mathbf{H}_{\|}^{\prime \prime(k)}\right) \cdot \mathbf{n}, \\
S_{j k} & =-\frac{1}{2} \int_{\partial \Gamma}\left(\mathbf{E}_{\|}^{\prime(j)} \times \mathbf{H}_{\|}^{\prime \prime(k)}\right) \cdot \mathbf{n}-\left(\mathbf{E}_{\|}^{\prime \prime(j)} \times \mathbf{H}_{\|}^{\prime(k)}\right) \cdot \mathbf{n} .
\end{aligned}
$$

which can be extracted from the experimental measurements of the boundary fields. Thus $W(\mathbf{E}, \mathbf{H})$ depends not only on the $n$ diagonal elements, $W_{j j}$, which physically represent the time-averaged power dissipation in each experiment, but also on the $n(n-1)$ elements $W_{j k}+W_{k j}$ and $S_{j k}-S_{k j}$, with $j>k$, which have no such physical interpretation. It makes sense to choose trial fields which are also linear combinations:

$$
\begin{gathered}
\underline{\mathbf{E}}^{\prime}=\sum_{j=1}^{n}\left(\lambda_{j} \underline{\mathbf{E}}^{(j)}\right)^{\prime}=\sum_{j=1}^{n} \lambda_{j}^{\prime} \underline{\mathbf{E}}^{(j)}-\lambda_{j}^{\prime \prime} \underline{\mathbf{E}}^{\prime \prime(j)}, \\
\underline{\mathbf{H}}^{\prime \prime}=\sum_{j=1}^{n}\left(\lambda_{j} \underline{\mathbf{H}}^{(j)}\right)^{\prime \prime}=\sum_{j=1}^{n} \lambda_{j}^{\prime} \underline{\mathbf{H}}^{\prime \prime(j)}+\lambda_{j}^{\prime \prime} \underline{\mathbf{H}}^{(j)} .
\end{gathered}
$$

Then $Y\left(\underline{\mathbf{E}}^{\prime}, \underline{\mathbf{H}}^{\prime \prime}\right) /(2 \omega)-W(\mathbf{E}, \mathbf{H})$ must be a positive quadratic form in the $2 n$ real variables $\lambda_{1}^{\prime}, \lambda_{1}^{\prime \prime}, \lambda_{2}^{\prime}, \lambda_{2}^{\prime \prime} \ldots \lambda_{n}^{\prime}, \lambda_{n}^{\prime \prime}$, and the positive semi-definiteness of the associated $2 n$ by $2 n$ matrix will give constaints on the functions $\boldsymbol{\varepsilon}(\mathbf{x})$ and $\boldsymbol{\mu}(\mathbf{x})$. 
We can also use the variational principle (5.18) for tomography purposes. When $\boldsymbol{\mu}$ is real and $\mathbf{j}=0$ the inequality

$$
W(\mathbf{E}, \mathbf{H}) \leq Y\left(\underline{\mathbf{E}}^{\prime}\right) /(2 \omega)
$$

applies. Often one knows in advance that the material inside $\Gamma$ is non-magnetic, so that $\boldsymbol{\mu}=\mathbf{I}$ inside $\Gamma$. Then from knowledge of $W(\mathbf{E}, \mathbf{H})$ (6.7) provides a constraint on the possible values of the electrical permittivity $\varepsilon$ for every suitable choice of the trial field $\underline{\mathbf{E}}^{\prime}$. On the other hand, suppose the medium inside $\Gamma$ was two phase with an unknown phase boundary and with two different values of $\boldsymbol{\mu}$ inside $\Gamma$. Then the variational principle (6.7) will be useless unless the choice of trial field is carefully correlated with the unknown phase boundary, so that the tangential component of $\boldsymbol{\mu}^{-1}\left(\nabla \times \underline{\mathbf{E}}^{\prime}\right)$ is continuous across the phase boundary.

When $\mathbf{j} \neq 0$ then we have the inequality

$$
W(\mathbf{E}, \mathbf{H}) \leq W(\underline{\mathbf{E}}, \underline{\mathbf{H}})
$$

where $W(\underline{\mathbf{E}}, \underline{\mathbf{H}})$ is given by $($ 5.16) and the trial fields $\underline{\mathbf{E}}$ and $\underline{\mathbf{H}}$ are required to have the same tangential values as, respectively, $\mathbf{E}$ and $\mathbf{H}$. Given measurements of the tangential values of $\mathbf{E}$ and $\mathbf{H}$, and trial fields $\underline{\mathbf{E}}$ and $\underline{\mathbf{H}}$ we expect that this inequality should provide a useful constraint on the possible values of $\varepsilon(\mathbf{x})$ and $\boldsymbol{\mu}(\mathbf{x})$.

\section{Acknowledgements}

The authors are grateful for support from the Université de Toulon et du Var, the University of Utah, and from the National Science Foundation through grant DMS-070978. The authors are grateful to Russell Richins for checking the manuscript, and to David Dobson for helpful discussions.

\section{References}

Altay, G. A. and M. C. Dökmeci 2004. Fundamental equations of certain electromagnetic-acoustic discontinuous fields in variational form. Continuum $\mathrm{Me}$ chanics and Thermodynamics 16:53-71.

Ammari, H. and H. Kang 2004. Reconstruction of small inhomogeneities from boundary measurements. Berlin: Springer-Verlag. x + 238 pp. ISBN 3-540-22483-1.

Ammari, H., M. S. Vogelius, and D. Volkov 2001. Asymptotic formulas for perturbations in the electromagnetic fields due to the presence of inhomogeneities of small diameter. II. The full Maxwell equations. Journal de Mathématiques Pures et Appliquées. Neuvième Série 80(8):769-814. 
Ávila, A., G. Griso, and B. Miara 2005. Bandes phononiques interdites en élasticité linéarisée. Comptes Rendus des Séances de l'Académie des Sciences. Série I. Mathématique 340:933-938.

Ben-Amoz, M. 1966. Variational principles in anisotropic and nonhomogeneous elastokinetics. Quarterly of Applied Mathematics XXIV(1):82-86.

Berryman, J. G. and R. V. Kohn 1990. Variational constraints for electrical-impedance tomography. Physical Review Letters 65(3):325-328.

Borcea, L. 1999. Asymptotic analysis of quasistatic transport in high contrast conductive media. SIAM Journal on Applied Mathematics 59(2):597-635.

Bouchitté, G. and D. Felbacq 2004. Homogenization near resonances and artificial magnetism from dielectrics. Comptes Rendus des Séances de l'Académie des Sciences. Série I. Mathématique 339:377-382.

Braides, A. 2002. Gamma-convergence for beginners. Oxford, United Kingdom: Oxford University Press. 230 pp. ISBN 9780198507840.

Cherkaev, A. V. and L. V. Gibiansky 1994. Variational principles for complex conductivity, viscoelasticity, and similar problems in media with complex moduli. Journal of Mathematical Physics 35(1):127-145.

Fannjiang, A. and G. Papanicolaou 1994. Convection enhanced diffusion for periodic flows. SIAM Journal on Applied Mathematics 54(2):333-408.

Felbacq, D. and G. Bouchitté 2005. Theory of mesoscopic magnetism in photonic crystals. Physical Review Letters 94:183902.

Gibiansky, L. V. and G. W. Milton 1993. On the effective viscoelastic moduli of twophase media. I. Rigorous bounds on the complex bulk modulus. Proceedings of the Royal Society of London. Series A, Mathematical and Physical Sciences 440(1908): 163-188.

Gibiansky, L. V., G. W. Milton, and J. G. Berryman 1999. On the effective viscoelastic moduli of two-phase media: III. Rigorous bounds on the complex shear modulus in two dimensions. Proceedings of the Royal Society of London. Series A, Mathematical and Physical Sciences 455(1986):2117-2149.

Gurtin, M. E. 1964. Variational principles in linear elastodynamics. Archive for Rational Mechanics and Analysis 16:34-50.

Hashin, Z. and S. Shtrikman 1962b. A variational approach to the theory of the effective magnetic permeability of multiphase materials. Journal of Applied Physics 33:31253131.

Hashin, Z. and S. Shtrikman 1962c. A variational approach to the theory of the elastic behavior of polycrystals. Journal of the Mechanics and Physics of Solids 10:343352. 
Hashin, Z. and S. Shtrikman 1962a. On some variational principles in anisotropic and non-homogeneous elasticity. Journal of the Mechanics and Physics of Solids 10: $335-342$.

Hashin, Z. and S. Shtrikman 1963. A variational approach to the theory of the elastic behavior of multiphase materials. Journal of the Mechanics and Physics of Solids 11:127-140.

Kohn, R. V. and M. Vogelius 1984. Determining conductivity by boundary measurements. Communications on pure and applied mathematics 37(3):289-298.

Liu, Z., C. T. Chan, and P. Sheng 2005. Analytic model of phononic crystals with local resonances. Physical Review B (Solid State) 71:014103.

Milton, G. W. 1990. On characterizing the set of possible effective tensors of composites: The variational method and the translation method. Communications on Pure and Applied Mathematics (New York) 43(1):63-125.

Milton, G. W. and J. G. Berryman 1997. On the effective viscoelastic moduli of twophase media. II. Rigorous bounds on the complex shear modulus in three dimensions. Proceedings of the Royal Society of London. Series A, Mathematical and Physical Sciences 453(1964):1849-1880.

Milton, G. W., M. Briane, and J. R. Willis 2006. On cloaking for elasticity and physical equations with a transformation invariant form. New Journal of Physics 8:248.

Milton, G. W. and J. R. Willis 2007. On modifications of Newton's second law and linear continuum elastodynamics. Proceedings of the Royal Society of London. Series A, Mathematical and Physical Sciences 463(2079):855-880.

Movchan, A. B. and S. Guenneau 2004. Split-ring resonators and localized modes. Physical Review B (Solid State) 70:125116.

Oden, J. T. and J. N. Reddy 1983. Variational Methods in Theoretical Mechanics. Berlin / Heidelberg / London / etc.: Springer-Verlag. xi + 309 pp. ISBN 0-38711917-5. LCCN QC808.03 1982.

Schoenberg, M. and P. N. Sen 1983. Properties of a periodically stratified acoustic half-space and its relation to a Biot fluid. The Journal of the Acoustical Society of America 73(1):61-67.

Sylvester, J. and G. Uhlmann 1987. A global uniqueness theorem for an inverse boundary value problem. Annals of Mathematics 125:153-169.

Tao, L. N. 1966. On variational principles for electromagnetic theory. Journal of Mathematical Physics 7(3):526-530.

Willis, J. R. 1981. Variational principles for dynamic problems for inhomogeneous elastic media. Wave Motion 3:1-11.

Willis, J. R. 1984. Variational principles and operator equations for electromagnetic waves in inhomogeneous media. Wave Motion 6:127-139. 